\title{
Absolute Whitehead torsion
}

\author{
ANDREW KORZENIEWSKI
}

\begin{abstract}
We refine the Whitehead torsion of a chain equivalence of finite chain complexes in an additive category $\mathbb{A}$ from an element of $\widetilde{K}_{1}^{\text {iso }}(\mathbb{A})$ to an element of the absolute group $K_{1}^{\text {iso }}(\mathbb{A})$. We apply this invariant to symmetric Poincaré complexes and identify it in terms of more traditional invariants. In the companion paper (joint with Ian Hambleton and Andrew Ranicki) this new invariant is applied to obtain the multiplicativity of the signature of fibre bundles mod 4 .
\end{abstract}

$57 \mathrm{Q} 10$

\section{Introduction}

The Whitehead torsion of a homotopy equivalence $f: X \rightarrow Y$ of finite $C W$ complexes is an element of the Whitehead group of $\pi=\pi_{1}(X)=\pi_{1}(Y)$

$$
\tau(f)=\tau(\widetilde{f}: C(\widetilde{X}) \rightarrow C(\widetilde{Y})) \in \mathrm{Wh}(\pi)=K_{1}(\mathbb{Z}[\pi]) /\{ \pm \pi\},
$$

with $\widetilde{f}$ the induced chain equivalence of based f.g. free cellular $\mathbb{Z}[\pi]$-module chain complexes. The Whitehead torsion of a finite $n$-dimensional Poincaré complex $X$ is

$$
\tau(X)=\tau\left([X] \cap-: C(\widetilde{X})^{n-*} \rightarrow C(\widetilde{X})\right) \in \mathrm{Wh}(\pi) .
$$

In this paper we extend the methods of Ranicki [7] to consider absolute Whitehead torsion invariants for homotopy equivalences of certain finite $C W$ complexes and finite Poincaré complexes, which take values in $K_{1}(\mathbb{Z}[\pi])$ rather than $\mathrm{Wh}(\pi)$. We shall also be extending the round $L$-theory of Hambleton-Ranicki-Taylor [2], which is the algebraic $L$-theory with absolute Whitehead torsion decorations. In the paper Hambleton-Korzeniewski-Ranicki [1] absolute Whitehead torsion in both algebraic $K$ - and $L$-theory will be applied to investigate the signatures of fibre bundles.

The absolute torsion of a finite contractible chain complex of finitely generated based $R$-modules $C$ is defined by

$$
\tau(C)=\tau\left(d+\Gamma: C_{\text {odd }} \rightarrow C_{\text {even }}\right) \in K_{1}(R) .
$$

for a chain contraction $\Gamma$; it is independent of the choice of $\Gamma$. The algebraic mapping cone of a chain equivalence of finite chain complexes of finitely generated based 
$R$-modules $f: C \rightarrow D$ is a contractible chain complex $\mathcal{C}(f)$. The naive absolute torsion $\tau(C(f)) \in K_{1}(R)$ only has good additive and composition formulae modulo $\operatorname{Im}\left(K_{1}(\mathbb{Z}) \rightarrow K_{1}(R)\right)$. Likewise, the naive definition of the torsion of an $n$-dimensional symmetric Poincaré complex $(C, \phi)$ (see Ranicki [5]) with $C$ a based f.g. free $R-$ module chain complex

$$
\tau(C, \phi)=\tau\left(\mathcal{C}\left(\phi_{0}: C^{n-*} \rightarrow C\right)\right)
$$

only has good cobordism and additivity properties in $\widetilde{K}_{1}(R)$. The Tate $\mathbf{Z}_{2}$-cohomology class

$$
\tau(C, \phi) \in \widehat{H}^{n}\left(\mathbf{Z}_{2} ; K_{1}(R)\right)
$$

may not be defined, and even if defined may not be a cobordism invariant.

In [7] Andrew Ranicki developed a theory of absolute torsion for chain equivalences of round chain complexes, that is chain complexes $C$ satisfying $\chi(C)=0$. This absolute torsion has a good composition formula but it is not additive, and for round Poincaré complexes $\tau\left(C, \phi_{0}\right)$ is not a cobordism invariant, contrary to the assertions of Ranicki $[8,(7.21),(7.22)]$.

There are two main aims of this paper, firstly to develop a more satisfactory definition of the absolute torsion of a chain equivalence with good additive and composition formulae and secondly to define an absolute torsion invariant of Poincaré complexes which behaves predictably under cobordism. Section 1 is devoted to the first of these aims. Following [7] we work in the more general context of an additive category $A$. The chief novelty here is the introduction of a signed chain complex; this is a pair $\left(C, \eta_{C}\right)$ where $C$ is a finite chain complex and $\eta_{C}$ is a "sign" term living in $K_{1}(\mathbb{A})$, which will be made precise in Section 1. We give definitions for the sum and suspension of two signed chain complexes, and we define the absolute torsion of a chain equivalence of signed chain complexes

$$
\tau^{\mathrm{NEW}}(f: C \rightarrow D) \in K_{1}^{\mathrm{iso}}(\mathbb{A}) .
$$

This gives us a definition of absolute torsion with good additive and composition formulae at the cost of making the definition more complicated by adding sign terms to the chain complexes. This definition is similar to the one given in [7], indeed if the chain complexes $C$ and $D$ are round and $\eta_{C}=\eta_{D}=0$ then the definition of the absolute torsion of a chain equivalence $f: C \rightarrow D$ is precisely that given in [7]. When working over a ring $R$ the absolute torsion defined here reduces to the usual torsion in $\widetilde{K}_{1}(R)$.

In Section 3 we work over a category with involution and define the dual of a signed chain complex. We can then define in Section 4 the absolute torsion of a symmetric 
Poincaré complex to be the absolute torsion of the chain equivalence $\phi_{0}: C^{n-*} \rightarrow C$. This new invariant is shown to be additive and to have good behaviour under round algebraic cobordism. Although we have to choose a sign $\eta_{C}$ in order to define the absolute torsion of $\phi_{0}$, we show that the absolute torsion is independent of this choice. In Section 6 we state a product formula for the absolute torsion and prove it for group rings.

It should be noted that the definitions in "Round $L$-Theory" [2] used the absolute torsion invariant of [7], [8], which is not a cobordism invariant. In Section 7 we show that the absolute torsion defined here may be used as the "correct" definition; in this case the statements in [2] are correct.

In Section 8 we investigate the absolute torsion of manifolds. This invariant is only defined when we pass to the reduced group $\widehat{H}^{n}\left(\mathbf{Z}_{2} ; K_{1}\left(\mathbf{Z}\left[\pi_{1} M\right]\right)\right)$ and we provide some examples. In Section 9 the "sign" term of the absolute torsion of a manifold is identified with more traditional invariants of a manifold such as the signature, the Euler characteristic and the semi-characteristic.

The forthcoming paper [1] will make extensive use of the invariants and techniques developed here. Section 2 and Section 5 develop the notion of the signed derived category which will be required by [1].

I would like to thank my supervisor Andrew Ranicki for his help and encouragement during the writing of this paper. I would also like to thank Ian Hambleton for many useful conversations and for carefully checking the computations.

\section{Absolute torsion of contractible complexes and chain equiv- alences}

In this section we introduce the absolute torsion of contractible complexes and chain equivalences and derive their basic properties. This closely follows [7] but without the assumption that the complexes are round $\left(\chi(C)=0 \in K_{0}(\mathbb{A})\right)$; we also develop the theory in the context of signed chain complexes which we will define in this section.

Let $\mathbb{A}$ be an additive category. Following [7] we make the following definition.

Definition 1 (1) The class group $K_{0}(\mathbb{A})$ has one generator $[M]$ for each object in A and relations:

(a) $[M]=\left[M^{\prime}\right]$ if $M$ is isomorphic to $\left[M^{\prime}\right]$

(b) $[M \oplus N]=[M]+[N]$ for objects $M, N$ in $\mathbb{A}$

(2) The isomorphism torsion group $K_{1}^{\text {iso }}(\mathbb{A})$ has one generator $\tau^{\text {iso }}(f)$ for each isomorphism $f: M \rightarrow N$ in $\mathbb{A}$, and relations: 
(a) $\tau^{\text {iso }}(g f)=\tau^{\text {iso }}(f)+\tau^{\text {iso }}(g)$ for isomorphisms $f: M \rightarrow N, g: N \rightarrow P$

(b) $\tau^{\text {iso }}\left(f \oplus f^{\prime}\right)=\tau^{\text {iso }}(f)+\tau^{\text {iso }}\left(f^{\prime}\right)$ for isomorphisms $f: M \rightarrow N$ and $f^{\prime}: M^{\prime} \rightarrow N^{\prime}$

\subsection{Sign terms}

The traditional torsion invariants are considered to lie in $\widetilde{K}_{1}^{\text {iso }}(\mathbb{A})$, a particular quotient of $K_{1}^{\text {iso }}(\mathbb{A})$ (defined below) in which the torsion of maps such as $\left(\begin{array}{ll}0 & 1 \\ 1 & 0\end{array}\right): C \oplus D \rightarrow D \oplus C$ are trivial. In absolute torsion we must consider such rearrangement maps; to this end we recall from [7] the following notation:

Definition 2 Let $C, D$ be finite chain complexes in $\mathbb{A}$.

(1) The suspension of $C$ is the chain complex $S C$ such that $S C_{r}=C_{r-1}$.

(2) The sign of two objects $M, N \in \mathbb{A}$ is the element

$$
\epsilon(X, Y):=\tau^{\text {iso }}\left(\left(\begin{array}{cc}
0 & 1_{N} \\
1_{M} & 0
\end{array}\right): M \oplus N \rightarrow N \oplus M\right) \in K_{1}^{\text {iso }}(\mathbb{A}) .
$$

The sign only depends on the stable isomorphism classes of $M$ and $N$ and satisfies

(a) $\epsilon\left(M \oplus M^{\prime}, N\right)=\epsilon(M, N)+\epsilon\left(M^{\prime}, N\right)$,

(b) $\epsilon(M, N)=-\epsilon(N, M)$,

(c) $\epsilon(M, M)=\tau^{\text {iso }}(-1: M \rightarrow M)$,

(d) $2 \epsilon(M, M)=0$.

We may extend $\epsilon$ to a morphism of abelian groups

$$
\epsilon: K_{0}(\mathbb{A}) \otimes K_{0}(\mathbb{A}) \rightarrow K_{1}^{\mathrm{iso}}(\mathbb{A}) ;([M],[N]) \mapsto \epsilon(M, N) .
$$

(3) The reduced isomorphism torsion group $\widetilde{K}_{1}^{\text {iso }}(\mathbb{A})$ is the quotient

$$
\widetilde{K}_{1}^{\text {iso }}(\mathbb{A}):=K_{1}^{\text {iso }}(\mathbb{A}) / \operatorname{Im}\left(\epsilon: K_{0}(\mathbb{A}) \otimes K_{0}(\mathbb{A}) \rightarrow K_{1}^{\text {iso }}(\mathbb{A})\right) .
$$

(4) The intertwining of $C$ and $D$ is the element defined by

$$
\beta(C, D):=\sum_{i>j}\left(\epsilon\left(C_{2 i}, D_{2 j}\right)-\epsilon\left(C_{2 i+1}, D_{2 j+1}\right)\right) \in K_{1}^{\text {iso }}(\mathbb{A}) .
$$

This depends only on the isomorphism classes of the chain complexes $C$ and $D$.

Example 3 The reader may find it useful to keep the following example in mind, as it is the most frequently occurring context. 
Let $R$ be an associative ring with 1 such that $\operatorname{rank}_{R}(M)$ is well-defined for f.g free modules $M$. We define $\mathbb{A}(R)$ to be the category of based f.g. $R$-modules. In this case the map $K_{0}(\mathbb{A}(R)) \rightarrow \mathbf{Z}$ given by $M \mapsto \operatorname{dim} M$ is an isomorphism. We have a forgetful functor

$$
K_{1}^{\text {iso }}(\mathbb{A}(R)) \rightarrow K_{1}(R) ; \tau^{\text {iso }}(f) \mapsto \tau(f),
$$

mapping elements of $K_{1}^{\text {iso }}(\mathbb{A}(R))$ to the more familiar $K_{1}(R)$ in the obvious way. In particular

$$
\operatorname{Im}\left(\epsilon: K_{0}(\mathbb{A}(R)) \otimes K_{0}(\mathbb{A}(R)) \rightarrow K_{1}(R)\right)=\{\tau( \pm 1)\}=\operatorname{Im}\left(K_{1}(\mathbf{Z}) \rightarrow K_{1}(R)\right)
$$

justifying the terminology of a "sign" term; the map is given explicitly for modules $M$ and $N$ by

$$
\epsilon(M, N)=\operatorname{rank}_{R}(M) \operatorname{rank}_{R}(N) \tau(-1) .
$$

We will make use of the notation

$$
\begin{aligned}
C_{\text {even }} & =C_{0} \oplus C_{2} \oplus C_{4} \oplus \cdots \\
C_{\text {odd }} & =C_{1} \oplus C_{3} \oplus C_{5} \oplus \cdots
\end{aligned}
$$

and as usual we define the Euler characteristic $\chi(C)$ as

$$
\chi(C)=\left[C_{\text {even }}\right]-\left[C_{\text {odd }}\right] \in K_{0}(\mathbb{A}) .
$$

We also recall from [7] (Proposition 3.4 and the remark above Proposition 3.3) the following relationships between the "sign" terms.

Lemma 4 Let $C, C^{\prime}, D, D^{\prime}$ be finite chain complexes over $\mathbb{A}$. Then

(1) $\beta(C, D)=\tau^{\text {iso }}\left((C \oplus D)_{\text {even }} \rightarrow C_{\text {even }} \oplus D_{\text {even }}\right)-\tau^{\text {iso }}\left((C \oplus D)_{\text {odd }} \rightarrow C_{\text {odd }} \oplus D_{\text {odd }}\right)$

(2) $\beta\left(C \oplus C^{\prime}, D\right)=\beta(C, D)+\beta\left(C^{\prime}, D\right)$

(3) $\beta\left(C, D \oplus D^{\prime}\right)=\beta(C, D)+\beta\left(C, D^{\prime}\right)$

(4) $\beta(C, D)-\beta(D, C)+\sum(-)^{r} \epsilon\left(C_{r}, D_{r}\right)=\epsilon\left(C_{\text {even }}, D_{\text {even }}\right)-\epsilon\left(C_{\text {odd }}, D_{\text {odd }}\right)$

(5) $\beta(S C, S D)=-\beta(C, D)$

(6) $\beta(S C, C)=\epsilon\left(C_{\text {odd }}, C_{\text {even }}\right)$.

\subsection{Signed chain complexes}

In order to make to formulae in this paper more concise we introduce the concept of a signed chain complex; this is a chain complex with an associated element in $\operatorname{Im}\left(\epsilon: K_{0}(\mathbb{A}) \otimes K_{0}(\mathbb{A}) \rightarrow K_{1}^{\text {iso }}(\mathbb{A})\right)$ which we refer to as the sign of the complex. We use this element in the definition of the absolute torsion invariants. 
Definition 5 (1) A signed chain complex is a pair $\left(C, \eta_{C}\right)$ where $C$ is a finite chain complex in $\mathbb{A}$ and $\eta_{C}$ an element of

$$
\operatorname{Im}\left(\epsilon: K_{0}(\mathbb{A}) \otimes K_{0}(\mathbb{A}) \rightarrow K_{1}^{\text {iso }}(\mathbb{A})\right)
$$

We will usually suppress mention of $\eta_{C}$ denoting such complexes as $C$.

(2) Given a signed chain complex $\left(C, \eta_{C}\right)$ we give the suspension of $C, S C$ the sign

$$
\eta_{S C}=-\eta_{C}
$$

(3) We define the sum signed chain complex of two signed chain complexes $\left(C, \eta_{C}\right)$, $\left(D, \eta_{D}\right)$ as $\left(C \oplus D, \eta_{C \oplus D}\right)$ where $C \oplus D$ is the usual based sum of two chain complexes and $\eta_{C \oplus D}$ defined by

$$
\eta_{C \oplus D}=\eta_{C}+\eta_{D}-\beta(C, D)+\epsilon\left(C_{\text {odd }}, \chi(D)\right)
$$

(it is easily shown that $\left.\eta_{(C \oplus D) \oplus E}=\eta_{C \oplus(D \oplus E)}\right)$.

\subsection{The absolute torsion of isomorphisms}

We now define the absolute torsion of a collection of isomorphisms $\left\{f_{r}: C_{r} \rightarrow D_{r}\right\}$ between two signed chain complexes. Note that the map $f$ need not be a chain isomorphism (ie $f d_{C}=d_{D} f$ need not hold). In the case where $f$ is a chain isomorphism the torsion invariant defined here will coincide with the definition of the absolute torsion of a chain equivalence given later.

Definition 6 The absolute torsion of a collection of isomorphisms $\left\{f_{r}: C_{r} \rightarrow D_{r}\right\}$ between the chain groups of signed chain complexes $C$ and $D$ is defined as

$$
\tau_{\text {iso }}^{\mathrm{NEW}}(f)=\sum_{r=-\infty}^{\infty}(-)^{r} \tau^{\mathrm{iso}}\left(f_{r}: C_{r} \rightarrow D_{r}\right)-\eta_{C}+\eta_{D} \in K_{1}^{\mathrm{iso}}(\mathbb{A}) .
$$

Lemma 7 We have the following properties of the absolute torsion of isomorphisms:

(1) The absolute torsion of isomorphisms is logarithmic, that is for isomorphisms $f: C \rightarrow D$ and $g: D \rightarrow E$.

$$
\tau_{\text {iso }}^{\mathrm{NEW}}(g f)=\tau_{\text {iso }}^{\mathrm{NEW}}(f)+\tau_{\text {iso }}^{\mathrm{NEW}}(g)
$$

(2) The absolute torsion of isomorphisms is additive, that is for isomorphisms $f: C \rightarrow D$ and $f^{\prime}: C^{\prime} \rightarrow D^{\prime}$

$$
\tau_{\text {iso }}^{\mathrm{NEW}}\left(f \oplus f^{\prime}\right)=\tau_{\text {iso }}^{\mathrm{NEW}}(f)+\tau_{\text {iso }}^{\mathrm{NEW}}\left(f^{\prime}\right)
$$


(3) The absolute torsion of the rearrangement isomorphism

$$
\left(\begin{array}{ll}
0 & 1 \\
1 & 0
\end{array}\right): C \oplus D \longrightarrow D \oplus C
$$

is $\epsilon(\chi(C), \chi(D)) \in K_{1}^{\mathrm{iso}}(\mathbb{A})$.

(4) The absolute torsion of the isomorphism

$$
\left(\begin{array}{ll}
0 & 1 \\
1 & 0
\end{array}\right): S(C \oplus D) \longrightarrow S C \oplus S D
$$

is $\epsilon(\chi(D), \chi(C)) \in K_{1}^{\text {iso }}(\mathbb{A})$.

Proof Parts 1 and 2 follow straight from the definitions, keeping in mind the fact that the sign terms $\epsilon$ and $\beta$ depend only on the isomorphism classes of their respective inputs. For part 3 we apply Lemma 4 part (4) to get

$$
\begin{aligned}
\tau_{\text {iso }}^{\mathrm{NEW}}(C \oplus D \rightarrow D \oplus C)= & \sum_{r=0}^{\infty}(-)^{r} \epsilon\left(C_{r}, D_{r}\right)-\eta_{C \oplus D}+\eta_{D \oplus C} \\
= & \sum_{r=0}^{\infty}(-)^{r} \epsilon\left(C_{r}, D_{r}\right)+\beta(C, D)-\beta(D, C) \\
& =\epsilon\left(C_{\text {even }}, D_{\text {even }}\right)-\epsilon\left(C_{\text {odd }}, D_{\text {odd }}\right) \\
& \left.-\epsilon\left(C_{\text {odd }}, \chi(D)\right)+\epsilon\left(D_{\text {odd }}, \chi(C)\right)\right) \\
= & \epsilon(\chi(C), \chi(D)) .
\end{aligned}
$$

For part 4

$$
\begin{aligned}
& \tau_{\text {iso }}^{\mathrm{NEW}}(S(C \oplus D) \rightarrow S C \oplus S D)=\eta_{S C \oplus S D}-\eta_{S(C \oplus D)} \\
& =-\beta(S C, S D)+\epsilon\left(C_{\text {even }}, \chi(S D)\right) \\
& -\beta(C, D)+\epsilon\left(C_{\text {odd }}, \chi(D)\right) \\
& =\epsilon(\chi(D), \chi(C)) \text {. }
\end{aligned}
$$

using Lemma 4 part (5).

\subsection{The absolute torsion of contractible complexes and short exact se- quences}

We recall from [7] the following:

Given a finite contractible chain complex over $\mathbb{A}$

$$
C: C_{n} \rightarrow \cdots \rightarrow C_{0}
$$


and a chain contraction $\Gamma: C_{r} \rightarrow C_{r+1}$ we may form the isomorphism

$$
d+\Gamma=\left(\begin{array}{cccc}
d & 0 & 0 & \cdots \\
\Gamma & d & 0 & \cdots \\
0 & \Gamma & d & \cdots \\
\vdots & \vdots & \vdots & \ddots
\end{array}\right): C_{\mathrm{odd}}=C_{1} \oplus C_{3} \oplus C_{5} \oplus \cdots \rightarrow C_{\mathrm{even}}=C_{0} \oplus C_{2} \oplus C_{4} \oplus \cdots
$$

The element $\tau^{\text {iso }}(d+\Gamma) \in K_{1}^{\text {iso }}(\mathbb{A})$ is independent of the choice of $\Gamma$ and is denoted $\tau(C)$ (following [7, Section 3]).

We define the absolute torsion of a contractible signed chain complex $C$ as

$$
\tau^{\mathrm{NEW}}(C)=\tau(C)+\eta_{C} \in K_{1}^{\mathrm{iso}}(\mathbb{A}) .
$$

Following [7] we give $\mathbb{A}$ the structure of an exact category by declaring that a sequence

$$
0 \rightarrow M \stackrel{i}{\rightarrow} M^{\prime \prime} \stackrel{j}{\rightarrow} M^{\prime} \rightarrow 0
$$

is exact if there exists a splitting morphism $k: M^{\prime} \rightarrow M^{\prime \prime}$ such that $j k=1: M^{\prime} \rightarrow M^{\prime}$ and the map

$$
\left(\begin{array}{ll}
i & k): M \oplus M^{\prime} \rightarrow M^{\prime \prime}
\end{array}\right.
$$

is an isomorphism.

Given a short exact sequence

$$
0 \rightarrow C \stackrel{i}{\rightarrow} C^{\prime \prime} \stackrel{j}{\rightarrow} C^{\prime} \rightarrow 0
$$

of signed chain complexes over $\mathbb{A}$, we may find a sequence of splitting morphisms $\left\{k: C_{r}^{\prime} \rightarrow C_{r}^{\prime \prime} \mid r \geq 0\right\}$ such that $j k=1: C_{r}^{\prime} \rightarrow C_{r}^{\prime}(r \geq 0)$ and each $(i \quad k): C_{r} \oplus C_{r}^{\prime} \rightarrow$ $C_{r}^{\prime \prime}(r \geq 0)$ is an isomorphism. The torsion of this collection of isomorphisms

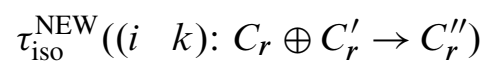

is independent of the choice of the $k_{r}$, so we may define the absolute torsion of a short exact sequence as

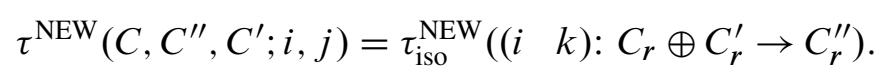

Lemma 8 We have the following properties of the absolute torsion of signed contractible complexes:

(1) Suppose we have a short exact sequence

$$
0 \rightarrow C \stackrel{i}{\rightarrow} C^{\prime \prime} \stackrel{j}{\rightarrow} C^{\prime} \rightarrow 0
$$


of contractible signed complexes. Then

$$
\tau^{\mathrm{NEW}}\left(C^{\prime \prime}\right)=\tau^{\mathrm{NEW}}(C)+\tau^{\mathrm{NEW}}\left(C^{\prime}\right)+\tau^{\mathrm{NEW}}\left(C, C^{\prime \prime}, C^{\prime} ; i, j\right) .
$$

(2) Let $C, C^{\prime}$ be contractible signed complexes. Then

$$
\tau^{\mathrm{NEW}}\left(C \oplus C^{\prime}\right)=\tau^{\mathrm{NEW}}(C)+\tau^{\mathrm{NEW}}\left(C^{\prime}\right) .
$$

\section{Proof}

(1) From [7, Proposition 3.3] we have that

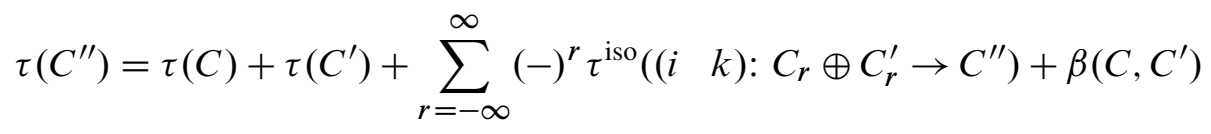

for some choice of splitting morphisms $\left\{k: C_{r}^{\prime} \rightarrow C_{r}^{\prime \prime} \mid r \geq 0\right\}$. By the definition of the absolute torsion of a short exact sequence and the definition of the sum torsion (noting that contractible complexes have $\chi(C)=0 \in K_{0}(\mathbb{A})$ ) we get

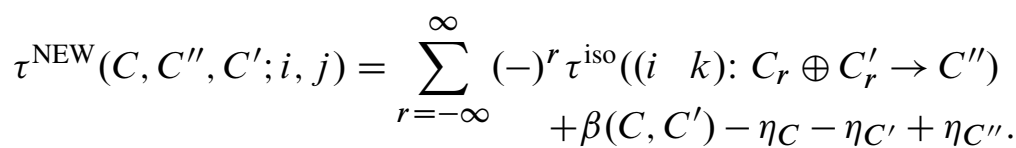

By comparing these two formulae and the definition of the absolute torsion of a contractible signed complex, the result follows.

(2) Apply the above to $C^{\prime \prime}=C \oplus C^{\prime}$.

\subsection{The absolute torsion of chain equivalences}

Sign Convention 9 We define the algebraic mapping cone of a chain map $f: C \rightarrow D$ as follows:

$$
d_{\mathcal{C}(f)}=\left(\begin{array}{cc}
d_{D} & (-)^{r+1} f \\
0 & d_{C}
\end{array}\right): \mathcal{C}(f)_{r}=D_{r} \oplus C_{r-1} \rightarrow \mathcal{C}(f)_{r-1}=D_{r-1} \oplus C_{r-2}
$$

We make $\mathcal{C}(f)$ into a signed complex by setting

$$
\eta_{\mathcal{C}(f)}=\eta_{D \oplus S C} .
$$

Lemma 10 The absolute torsion of a chain isomorphism $f: C \rightarrow D$ of signed chain complexes satisfies

$$
\tau_{\text {iso }}^{\mathrm{NEW}}(f)=\tau^{\mathrm{NEW}}(\mathcal{C}(f)) .
$$


Proof In the case of an isomorphism we may choose the chain contraction for $\mathcal{C}(f)$ to be

$$
\Gamma_{\mathcal{C}(f)}=\left(\begin{array}{cc}
0 & 0 \\
(-)^{r} f^{-1} & 0
\end{array}\right): \mathcal{C}(f)_{r} \rightarrow \mathcal{C}(f)_{r+1}
$$

We have a commutative diagram

$$
\begin{aligned}
& \left(D_{1} \oplus C_{0}\right) \oplus\left(D_{3} \oplus C_{2}\right) \oplus \cdots \stackrel{\left(d_{\mathcal{C}(f)}+\Gamma_{\mathcal{C}(f)}\right)}{\longrightarrow} D_{0} \oplus\left(D_{2} \oplus C_{1}\right) \oplus\left(D_{4} \oplus C_{3}\right) \oplus \cdots \\
& \downarrow\left(\begin{array}{cccc}
f & d_{D} & 0 & \ldots \\
0 & -f^{-1} & d_{C} & \ldots \\
0 & 0 & f & \ldots \\
\vdots & \vdots & \vdots & \ddots
\end{array}\right) \downarrow \\
& C_{0} \oplus D_{1} \oplus C_{2} \oplus D_{3} \oplus \cdots \longrightarrow D_{0} \oplus C_{1} \oplus D_{2} \oplus C_{3} .
\end{aligned}
$$

The torsion of the upper map is $\tau^{\text {iso }}(\mathcal{C}(f))$, the torsion of the lower isomorphism is $\sum_{r=-\infty}^{\infty}(-)^{r} \tau^{\text {iso }}\left(f_{r}: C_{r} \rightarrow D_{r}\right)+\epsilon\left(C_{\text {odd }}, C_{\text {odd }}\right)$ and the difference between the torsions of the downward maps is $\sum_{r=-\infty}^{\infty}(-)^{r} \epsilon\left(C_{r}, C_{r-1}\right)$ (using the fact that $C_{r} \cong$ $\left.D_{r}\right)$. Hence

$$
\begin{aligned}
\tau^{\mathrm{NEW}}(\mathcal{C}(f))= & \tau^{\mathrm{iso}}(\mathcal{C}(f))+\eta_{\mathcal{C}(f)} \\
= & \sum_{r=-\infty}^{\infty}(-)^{r} \tau^{\mathrm{iso}}\left(f_{r}: C r \rightarrow D_{r}\right)-\sum_{r=-\infty}^{\infty}(-)^{r} \epsilon\left(C_{r}, C_{r-1}\right) \\
& \quad-\beta(C, S C)+\epsilon\left(C_{\text {odd }}, \chi(S C)\right)+\epsilon\left(C_{\text {odd }}, C_{\text {odd }}\right)-\eta_{C}+\eta_{D} \\
& =\sum_{r=-\infty}^{\infty}(-)^{r} \tau^{\text {iso }}\left(f_{r}: C_{r} \rightarrow D_{r}\right)-\eta_{C}+\eta_{D} \\
& =\tau_{\text {iso }}^{\text {NEW }}(f)
\end{aligned}
$$

(using the formulae of Lemma 4).

We can now give a definition of the absolute torsion of a chain equivalence $f: C \rightarrow D$ which coincides with the previous definition in the case when $f$ is a chain isomorphism.

Definition 11 We define the absolute torsion of a chain equivalence of signed chain complexes $f: C \rightarrow D$ as

$$
\tau^{\mathrm{NEW}}(f)=\tau^{\mathrm{NEW}}(\mathcal{C}(f)) \in K_{1}^{\mathrm{iso}}(\mathbb{A}) .
$$

In the case where $f$ is a chain isomorphism the above lemma shows that this definition of the torsion agrees with that given in Definition 6. 
The following lemma explicitly describes how to compute the absolute torsion of a chain equivalence. It is intended primarily to allow readers already familiar with the absolute torsion of [7] to form a direct comparison with the new definition.

Lemma 12 The absolute torsion of a chain equivalence of chain complexes with torsion $f: C \rightarrow D$ is

$$
\tau^{\mathrm{NEW}}(f)=\tau(\mathcal{C}(f))-\beta(D, S C)-\epsilon\left(D_{\text {odd }}, \chi(C)\right)+\eta_{D}-\eta_{C} \in K_{1}(\mathbb{A})
$$

(cf definition of torsion [7, pp 223 and 226]. The two definitions coincide if both $\chi(C)$ and $\chi(D)$ are even and $\left.\eta_{C}=\eta_{D}\right)$.

Proof The proof is simply a matter of unravelling definitions.

We have the following properties of the absolute torsion of chain equivalences.

\section{Proposition 13}

(1) Let $f: C \rightarrow D$ and $g: D \rightarrow E$ be chain equivalences of signed chain complexes in $\mathbb{A}$, then

$$
\tau^{\mathrm{NEW}}(g f)=\tau^{\mathrm{NEW}}(f)+\tau^{\mathrm{NEW}}(g) \in K_{1}^{\mathrm{iso}}(\mathbb{A}) .
$$

(2) Suppose $f: C \rightarrow D$ is a chain equivalence of contractible signed chain complexes. Then

$$
\tau^{\mathrm{NEW}}(f)=\tau^{\mathrm{NEW}}(D)-\tau^{\mathrm{NEW}}(C) \in K_{1}^{\mathrm{iso}}(\mathbb{A}) .
$$

(3) The absolute torsion $\tau^{\mathrm{NEW}}(f)$ is a chain homotopy invariant of $f$.

(4) Suppose we have a commutative diagram of chain maps as follows where the rows are exact and the vertical maps are chain equivalences



Then

$$
\begin{array}{r}
\tau^{\mathrm{NEW}}(b)=\tau^{\mathrm{NEW}}(a)+\tau^{\mathrm{NEW}}(c)-\tau^{\mathrm{NEW}}(A, B, C ; i, j) \\
+\tau^{\mathrm{NEW}}\left(A^{\prime}, B^{\prime}, C^{\prime} ; i^{\prime}, j^{\prime}\right) \in K_{1}^{\mathrm{iso}}(\mathbb{A}) .
\end{array}
$$

(5) The torsion of a sum $f \oplus f^{\prime}: C \oplus C^{\prime} \rightarrow D \oplus D^{\prime}$ is given by

$$
\tau^{\mathrm{NEW}}\left(f \oplus f^{\prime}\right)=\tau^{\mathrm{NEW}}(f)+\tau^{\mathrm{NEW}}\left(f^{\prime}\right) \in K_{1}^{\mathrm{iso}}(\mathbb{A}) .
$$


(6) Suppose we have a short exact sequence

$$
0 \rightarrow A \stackrel{f}{\rightarrow} B \stackrel{g}{\rightarrow} C \rightarrow 0
$$

where $C$ is a contractible complex and $f$ is a chain equivalence. Then

$$
\tau^{\mathrm{NEW}}(f)=\tau^{\mathrm{NEW}}(A, B, C ; f, g)+\tau^{\mathrm{NEW}}(C) .
$$

Proof The proofs of these follow those in [7, Proposition 4.2 and Proposition 4.4] modified where appropriate.

(1) We denote by $\Omega C$ the chain complex defined by

$$
d_{\Omega C}=d_{C}: \Omega C_{r}=C_{r+1} \rightarrow \Omega C_{r-1}=C_{r} .
$$

We define a chain map

$$
h: \Omega \mathcal{C}(g) \rightarrow \mathcal{C}(f)
$$

by

$$
\left(\begin{array}{cc}
0 & -1 \\
0 & 0
\end{array}\right): \Omega \mathcal{C}(g)_{r}=E_{r+1} \oplus D_{r} \rightarrow \mathcal{C}(f)_{r}=D_{r} \oplus C_{r-1} .
$$

The algebraic mapping cone $\mathcal{C}(h)$ fits into the short exact sequences

$$
\begin{gathered}
0 \rightarrow \mathcal{C}(f) \stackrel{i}{\rightarrow} \mathcal{C}(h) \stackrel{j}{\rightarrow} \mathcal{C}(g) \rightarrow 0 \\
0 \rightarrow \mathcal{C}(g f) \stackrel{i^{\prime}}{\rightarrow} \mathcal{C}(h) \stackrel{j^{\prime}}{\rightarrow} \mathcal{C}\left(-1_{D}: D \rightarrow D\right) \rightarrow 0
\end{gathered}
$$

where

$$
\begin{aligned}
& i=\left(\begin{array}{l}
1 \\
0
\end{array}\right): \mathcal{C}(f)_{r} \rightarrow \mathcal{C}(h)_{r}=\mathcal{C}(f)_{r} \oplus \mathcal{C}(g)_{r} \\
& j=\left(\begin{array}{ll}
0 & 1
\end{array}\right): \mathcal{C}(h)_{r}=\mathcal{C}(f)_{r} \oplus \mathcal{C}(g)_{r} \rightarrow \mathcal{C}(g)_{r} \\
& i^{\prime}=\left(\begin{array}{ll}
0 & 0 \\
0 & 1 \\
1 & 0 \\
0 & f
\end{array}\right): \mathcal{C}(g f)_{r}=E_{r} \oplus S C_{r} \rightarrow \mathcal{C}(h)_{r}=D_{r} \oplus S C_{r} \oplus E_{r} \oplus S D_{r} \\
& j^{\prime}=\left(\begin{array}{cccc}
1 & 0 & 0 & 0 \\
0 & -f & 0 & 1
\end{array}\right): \mathcal{C}(h)_{r}=D_{r} \oplus S C_{r} \oplus E_{r} \oplus S D_{r} \rightarrow \mathcal{C}\left(-1_{D}\right)_{r}=D_{r} \oplus S D_{r} .
\end{aligned}
$$

Applying Lemma 8 part (1) to the first short exact sequence (1-1) we have

$$
\tau^{\mathrm{NEW}}(h)=\tau^{\mathrm{NEW}}(f)+\tau^{\mathrm{NEW}}(g) .
$$


Notice that

$$
\begin{aligned}
\tau_{\text {iso }}^{\mathrm{NEW}}\left(\left(i^{\prime} k^{\prime}\right)\right)= & \tau_{\text {iso }}^{\mathrm{NEW}}\left(\left(\begin{array}{llll}
0 & 0 & 1 & 0 \\
0 & 1 & 0 & 0 \\
1 & 0 & 0 & 0 \\
0 & f & 0 & 1
\end{array}\right): \mathcal{C}(g f)_{r} \oplus \mathcal{C}\left(-1_{D}\right)_{r} \rightarrow \mathcal{C}(h)\right) \\
= & \tau_{\text {iso }}^{\mathrm{NEW}}(D \oplus S C \rightarrow S C \oplus D) \\
& +\tau_{\text {iso }}^{\mathrm{NEW}}(E \oplus S C \rightarrow S C \oplus E) \\
& +\tau_{\text {iso }}^{\mathrm{NEW}}(D \oplus E \rightarrow E \oplus D) \\
= & \epsilon(\chi(D), \chi(D))
\end{aligned}
$$

(using the results of Lemma 7, the fact that $\chi(C)=\chi(D)=\chi(E)$ and that $f$ has no effect on the torsion). We also see that $\tau^{\mathrm{NEW}}\left(\mathcal{C}\left(-1_{D}\right)\right)=\tau_{\text {iso }}^{\mathrm{NEW}}\left(-1_{D}\right)=$ $\epsilon(\chi(D), \chi(D))$. Applying these two expressions and Lemma 8 part (1) to the second exact sequence (1-2) we see that

$$
\tau^{\mathrm{NEW}}(g f)=\tau^{\mathrm{NEW}}(h)
$$

and comparison with (1-3) yields the result.

(2) By construction we have $\mathcal{C}(0 \stackrel{0}{\rightarrow} D)=D$ and hence

$$
\tau^{\mathrm{NEW}}(0 \stackrel{0}{\rightarrow} D)=\tau^{\mathrm{NEW}}(D) .
$$

Applying this and the composition formula (part 1) to the composition

$$
0 \stackrel{0}{\rightarrow} C \stackrel{f}{\rightarrow} D
$$

yields the result.

(3) A chain homotopy

$$
g: f \simeq f^{\prime}: C \rightarrow D
$$

gives rise to an isomorphism

$$
\left(\begin{array}{cc}
1 & (-)^{r} g \\
0 & 1
\end{array}\right): \mathcal{C}(f)=D \oplus S C \rightarrow \mathcal{C}\left(f^{\prime}\right)=D \oplus S C
$$

which has trivial torsion. Using part 2

$$
0=\tau^{\mathrm{NEW}}\left(\mathcal{C}\left(f^{\prime}\right)\right)-\tau^{\mathrm{NEW}}(\mathcal{C}(f))
$$

the result follows.

(4) We choose splitting morphisms $\left\{k: C_{r} \rightarrow B_{r} \mid r \geq 0\right\}$ and $\left\{k^{\prime}: C_{r}^{\prime} \rightarrow B_{r}^{\prime} \mid r \geq 0\right\}$. We have the following short exact sequence of mapping cones:

$$
0 \rightarrow \mathcal{C}(a) \stackrel{\left(\begin{array}{ll}
i^{\prime} & 0 \\
0 & i
\end{array}\right)}{\longrightarrow} \mathcal{C}(b) \stackrel{\left(\begin{array}{ll}
j^{\prime} & 0 \\
0 & j
\end{array}\right)}{\longrightarrow} \mathcal{C}(c) \rightarrow 0
$$


We note that

$$
\begin{aligned}
& \tau^{\mathrm{NEW}}\left(\mathcal{C}(a), \mathcal{C}(b), \mathcal{C}(c) ;\left(\begin{array}{c}
i^{\prime} \\
i
\end{array}\right) ;\left(\begin{array}{c}
i^{\prime} \\
i
\end{array}\right)\right) \\
& =\tau_{\text {iso }}^{\mathrm{NEW}}\left(\left(\begin{array}{cccc}
i^{\prime} & 0 & k^{\prime} & 0 \\
0 & i & 0 & k
\end{array}\right): A^{\prime} \oplus S A \oplus C^{\prime} \oplus S C \rightarrow B^{\prime} \oplus B\right) \\
& =\tau_{\text {iso }}^{\mathrm{NEW}}\left(\left(\begin{array}{ccccc}
i^{\prime} & k^{\prime} & 0 & 0 \\
0 & 0 & i & k
\end{array}\right): A^{\prime} \oplus C^{\prime} \oplus S A \oplus S C \rightarrow B^{\prime} \oplus B\right) \\
& +\tau_{\text {iso }}^{\mathrm{NEW}}\left(S A \oplus C^{\prime} \rightarrow C^{\prime} \oplus S A\right)
\end{aligned}
$$

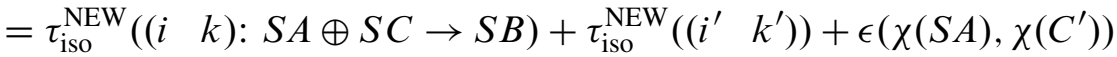

$$
\begin{aligned}
& =\tau_{\text {iso }}^{\mathrm{NEW}}((i \quad k): S(A \oplus C) \rightarrow S B)+\tau_{\text {iso }}^{\mathrm{NEW}}\left(\left(i^{\prime} \quad k^{\prime}\right)\right) \\
& +\tau_{\text {iso }}^{\mathrm{NEW}}(S A \oplus S C \rightarrow S(A \oplus C))+\epsilon(\chi(C), \chi(A))
\end{aligned}
$$



$$
\begin{aligned}
& -\epsilon(\chi(C), \chi(A))+\epsilon(\chi(C), \chi(A))
\end{aligned}
$$



$$
\begin{aligned}
& =\tau^{\mathrm{NEW}}\left(A^{\prime}, B^{\prime}, C^{\prime} ; i^{\prime}, j^{\prime}\right)-\tau^{\mathrm{NEW}}(A, B, C ; i, j) \text {. }
\end{aligned}
$$

The result now follows from applying Lemma 8 part (1) to the short exact sequence above.

(5) Applying the result for a commutative diagram of short exact sequences (part (4)) with $a=f: C \rightarrow D, c=f^{\prime}: C^{\prime} \rightarrow D^{\prime}$ and $b=f \oplus f^{\prime}: C \oplus C^{\prime} \rightarrow D \oplus D^{\prime}$ yields the result.

(6) We have a commutative diagram with short exact rows:

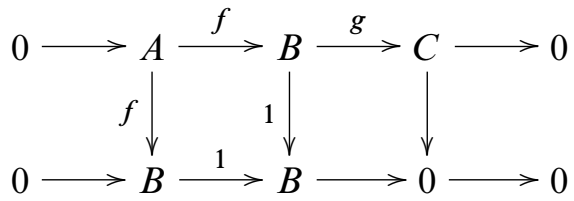

The result follows by applying part (4) to the above diagram.

\subsection{Applications to topology and examples of use}

Let $X$ be a connected finite $C W$-complex. We may form the cellular chain complex of the universal cover of $X$ as a complex $C(\tilde{X})$ over the fundamental group ring $\mathbf{Z}\left[\pi_{1} X\right]$; we may further make $C(\tilde{X})$ into a signed complex with an arbitrary choice of $\eta_{C(\tilde{X})}$. For a cellular homotopy equivalence $f: X \rightarrow X$ we have an associated chain equivalence $f_{*}: C(\tilde{X}) \rightarrow C(\tilde{X})$; we can make $C(\tilde{X})$ into a signed chain complex by choosing some $\eta_{C(\tilde{X})}$ and defining the torsion of $f$ to be

$$
\tau^{\mathrm{NEW}}(f):=\tau^{\mathrm{NEW}}\left(f_{*}: C(\tilde{X}) \rightarrow C(\tilde{X})\right) \in K_{1}\left(\mathbf{Z}\left[\pi_{1} X\right]\right)
$$


which is independent of the choice of $\eta_{C(\tilde{X})}$. We now give some examples.

(1) The torsion of the identity map of any connected $C W$-complex is trivial.

(2) Let $X=\mathbb{C P}^{2}$; we choose homogeneous coordinates $(x: y: z)$ and we give $X$ a $C W$-structure as follows:

$$
\begin{array}{ll}
0-\text { cell } & (1: 0: 0) \\
\text { 2-cell } & \left(z_{1}: 1: 0\right) \\
\text { 4-cell } & \left(z_{1}: z_{2}: 1\right)
\end{array}
$$

Let $f: \mathbb{C P}^{2} \rightarrow \mathbb{C P}^{2}$ be the cellular self-homeomorphism given by complex conjugation in all three coordinates, that is,

$$
f:(x: y: z) \mapsto(\bar{x}: \bar{y}: \bar{z}) .
$$

This map preserves the orientation of the 0-cell and 4-cell, and it reverses the orientation of the 2-cell. Hence $\tau^{\mathrm{NEW}}(f)=\tau(-1)$. In Corollary 38 we show that for any orientation preserving self-homeomorphism $g$ of a simply connected manifold of dimension $4 k+2$, that $\tau^{\mathrm{NEW}}(g)=0$. This example shows that for self-homeomorphism $f$ of a $4 k$-dimensional manifold it is possible for $\tau^{\mathrm{NEW}}(f) \neq 0$.

\section{The signed derived category}

The forthcoming paper [1] will require the use of the signed derived category $\operatorname{SD}(\mathbb{A})$. In this section we define $\mathbb{S} \mathbb{D}(\mathbb{A})$ and prove some basic properties.

Definition 14 The signed derived category $\mathbb{S} \mathbb{D}(\mathbb{A})$ is the category with objects finite signed chain complexes in $\mathbb{A}$ and morphisms chain homotopy classes of chain maps between such complexes.

\section{Proposition 15}

(i) The Euler characteristic defines a surjection

$$
\chi: K_{0}(\operatorname{SDD}(\mathbb{A})) \rightarrow K_{0}(\mathbb{A}) ;\left[C, \eta_{C}\right] \mapsto \chi(C)=\sum_{r=-\infty}^{\infty}(-)^{r}\left[C_{r}\right]
$$

(ii) Isomorphism torsion defines a forgetful map

$$
i_{*}: K_{1}^{\text {iso }}(\mathbb{S} \mathbb{D}(\mathbb{A})) \rightarrow K_{1}^{\text {iso }}(\mathbb{A}) ; \tau^{\text {iso }}(f) \mapsto\left[\tau^{\mathrm{NEW}}(f)\right]=\tau^{\mathrm{NEW}}(f)
$$


which is a surjection split by the injection $K_{1}^{\text {iso }}(\mathbb{A}) \rightarrow K_{1}^{\text {iso }}(\mathbb{S D}(\mathbb{A})) ; \tau^{\text {iso }}(f: A \rightarrow B) \mapsto \tau^{\text {iso }}(f:(A, 0) \rightarrow(B, 0))$.

where $(A, 0)$ (respectively $(B, 0))$ is the signed chain complex which is $A$ (respectively $B$ ) in dimension 0 , trivial elsewhere and with trivial sign.

(iii) The diagram

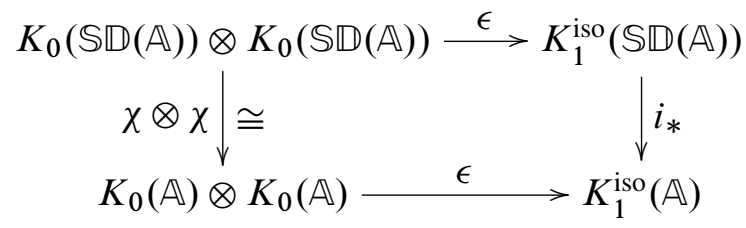

commutes, that is the sign of objects $\left(C, \eta_{C}\right),\left(D, \eta_{D}\right)$ in $\mathbb{S} \mathbb{D}(\mathbb{A})$ has image

$$
i_{*} \epsilon\left(\left(C, \eta_{C}\right),\left(D, \eta_{D}\right)\right)=\epsilon(\chi(C), \chi(D)) \in K_{1}^{\text {iso }}(\mathbb{A}) .
$$

\section{Proof}

(i) A short exact sequence $0 \rightarrow C \rightarrow D \rightarrow E \rightarrow 0$ of finite chain complexes in $\mathbb{A}$ determines a relation

$$
\left[C, \eta_{C}\right]-\left[D, \eta_{D}\right]+\left[E, \eta_{E}\right]=0 \in K_{0}(\mathbb{S D D}(\mathbb{A}))
$$

for any signs $\eta_{C}, \eta_{D}, \eta_{E}$.

(ii) By construction.

(iii) The sign

$\epsilon\left(\left(C, \eta_{C}\right),\left(D, \eta_{D}\right)\right)=\tau^{\mathrm{NEW}}\left(\left(\begin{array}{ll}0 & 1 \\ 1 & 0\end{array}\right):\left(C, \eta_{C}\right) \oplus\left(D, \eta_{D}\right) \rightarrow\left(D, \eta_{D}\right) \oplus\left(C, \eta_{C}\right)\right) \in K_{1}^{\text {iso }}(\mathbb{S} \mathbb{D}(\mathbb{A}))$

has image

$$
\begin{aligned}
i_{*} \epsilon\left(\left(C, \eta_{C}\right),\left(D, \eta_{D}\right)\right) & =\tau^{\mathrm{NEW}}\left(\left(\begin{array}{ll}
0 & 1 \\
1 & 0
\end{array}\right): C \oplus D \rightarrow D \oplus C\right) \\
& =\epsilon(\chi(C), \chi(D)) \in K_{1}^{\mathrm{iso}}(\mathbb{A}) .
\end{aligned}
$$

by Lemma 7 part 3 .

\section{Duality properties of absolute torsion}

In this section we extend the notion of absolute torsion to encompass dual objects and dual maps. We now work over an additive category with involution (defined below) and introduce the notion of a dual signed complex $C^{n-*}$ (also defined below). We prove the following results. 


\section{Proposition 16}

(1) Let $C$ be a contractible signed complex. Then

$$
\tau^{\mathrm{NEW}}\left(C^{n-*}\right)=(-)^{n+1} \tau^{\mathrm{NEW}}(C)^{*} \in K_{1}^{\mathrm{iso}}(\mathbb{A}) .
$$

(2) Let $f: C \rightarrow D$ be a chain equivalence of signed chain complexes. Then

$$
\tau^{\mathrm{NEW}}\left(f^{n-*}: C^{n-*} \rightarrow D^{n-*}\right)=(-)^{n} \tau^{\mathrm{NEW}}(f)^{*} \in K_{1}^{\mathrm{iso}}(\mathbb{A}) .
$$

(3) Let $f: C^{n-*} \rightarrow D$ be a chain equivalence of signed chain complexes. Then the chain equivalence $T f: D^{n-*} \rightarrow C$ (defined below) satisfies

$$
\tau^{\mathrm{NEW}}\left(T f: D^{n-*} \rightarrow C\right)=(-)^{n} \tau^{\mathrm{NEW}}(f)^{*}+\frac{n}{2}(n+1) \epsilon(\chi(C), \chi(C)) \in K_{1}^{\text {iso }}(\mathbb{A}) .
$$

The rest of this section will be concerned with defining these concepts and proving Proposition 16.

Following Ranicki [8] we define an involution on an additive category $\mathbb{A}$ to be a contravariant functor

$$
*: \mathbb{A} \rightarrow \mathbb{A} ; M \rightarrow M^{*},(f: M \rightarrow N) \rightarrow\left(f^{*}: N^{*} \rightarrow M^{*}\right)
$$

together with a natural equivalence

$$
e: \operatorname{id}_{\mathbb{A}} \rightarrow * *: \mathbb{A} \rightarrow \mathbb{A} ; M \rightarrow\left(e(M): M \rightarrow M^{* *}\right)
$$

such that for any object $M$ of A

$$
e\left(M^{*}\right)=\left(e(M)^{-1}\right)^{*}: M^{*} \rightarrow M^{* * *} .
$$

An involution on $\mathbb{A}$ induces an involution on $K_{0}(\mathbb{A})$ and $K_{1}^{\text {iso }}(\mathbb{A})$ in the obvious way. We can easily deduce the following properties of the sign terms with respect to the involution

(1) $\chi\left(C^{n-*}\right)=(-)^{n} \chi(C)^{*}$

(2) $\epsilon\left(A^{*}, B^{*}\right)=\epsilon(B, A)^{*}$

(3) $\beta\left(C^{n-*}, D^{n-*}\right)=(-)^{n} \beta(D, C)^{*}$.

Throughout the rest of this chapter $\mathbb{A}$ is an additive category with involution.

Sign Convention 17 Given an $n$-dimensional chain complex

$$
C: C_{n} \stackrel{d_{C}}{\longrightarrow} C_{n-1} \stackrel{d_{C}}{\longrightarrow} C_{n-2} \stackrel{d_{C}}{\longrightarrow} \cdots \stackrel{d_{C}}{\longrightarrow} C_{0}
$$


in $\mathbb{A}$ we may form the dual chain complex

$$
C^{n-*}: C^{0} \stackrel{d_{C^{n-*}}}{\longrightarrow} C^{1} \stackrel{d_{C^{n-*}}}{\longrightarrow} \cdots \stackrel{d_{C^{n-*}}}{\longrightarrow} C^{n}
$$

with the $r$ th object given by $C^{n-r}=C_{n-r}^{*}$ and the differentials given by

$$
d_{C^{n-*}}=(-)^{r} d_{C}^{*}: C^{n-r} \rightarrow C^{n-r+1} .
$$

We define the sign term

$$
\alpha_{n}(C)=\sum_{r \equiv n+2, n+3(\bmod 4)} \epsilon\left(C^{r}, C^{r}\right) . \in K_{1}^{\mathrm{iso}}(\mathbb{A}) .
$$

Given a signed chain complex $\left(C, \eta_{C}\right)$ we define the dual signed chain complex $\left(C^{n-*}, \eta_{C^{n-*}}\right)$ by

$$
\eta_{C^{n-*}}:=(-)^{n+1} \eta_{C}^{*}+(-)^{n+1} \beta(C, C)^{*}+\alpha_{n}(C) \in K_{1}^{\text {iso }}(\mathbb{A}) .
$$

We now compute the absolute torsion of various useful chain equivalences.

Lemma 18 Let $C$ and $D$ be signed chain complexes over $A$.

(1) For chain isomorphisms $f: C \rightarrow D$ we have

$$
\tau^{\mathrm{NEW}}\left(f^{n-*}: D^{n-*} \rightarrow C^{n-*}\right)=(-)^{n} \tau^{\mathrm{NEW}}(f)^{*} .
$$

(2) $\tau^{\mathrm{NEW}}\left((C \oplus D)^{n-*} \rightarrow C^{n-*} \oplus D^{n-*}\right)= \begin{cases}0 & \text { for } n \text { even } \\ \epsilon(\chi(C), \chi(D))^{*} & \text { for } n \text { odd. }\end{cases}$

(3) $\tau^{\mathrm{NEW}}\left(1: C^{n-*} \rightarrow(S C)^{n+1-*}\right)=0$.

(4) $\tau^{\mathrm{NEW}}\left((-1)^{r}: C_{r}^{n+1-*} \rightarrow S\left(C^{n-*}\right)_{r}\right)=0$.

(5) $\tau^{\mathrm{NEW}}\left((-1)^{(n+1) r}:\left(C^{n-*}\right)_{r}^{n-*} \rightarrow C_{r}\right)=\frac{n}{2}(n+1) \epsilon(\chi(C), \chi(C))^{*}$.

Proof Part 1 follows straight from the definitions. For part 2,

$$
\begin{aligned}
\tau^{\mathrm{NEW}} & \left((C \oplus D)^{n-*} \rightarrow C^{n-*} \oplus D^{n-*}\right) \\
& =\eta_{C^{n-*} \oplus D^{n-*}-\eta_{(C \oplus D)^{n-*}}} \\
& =\epsilon\left(\chi\left(C^{n-*}\right),\left(D^{n-*}\right)_{\text {even }}\right)+(-)^{n} \epsilon\left(\chi(C), D_{\text {even }}\right)^{*} .
\end{aligned}
$$

The result follows after considering the odd and even cases.

Part 3 follows straight from the definitions. For part 4,

$$
\begin{aligned}
\tau^{\mathrm{NEW}} & \left((-1)^{r}: C_{r}^{n+1-*} \rightarrow S\left(C^{n-*}\right)_{r}\right) \\
& =\eta_{S\left(C^{n-*}\right)-\eta_{C} n+1-*}+\sum_{r \equiv n(\bmod 2)} \epsilon\left(C_{r}, C_{r}\right)^{*} \\
& =\alpha_{n+1}(C)+\alpha_{n}(C)+\sum_{r \equiv n(\bmod 2)} \epsilon\left(C_{r}, C_{r}\right)^{*} \\
& =0 .
\end{aligned}
$$


For part 5,

$$
\begin{aligned}
& \tau^{\mathrm{NEW}}\left((-1)^{(n+1) r}:\left(C^{n-*}\right)_{r}^{n-*} \rightarrow C_{r}\right) \\
& =\eta_{C}-\eta_{\left(C^{n-*}\right)^{n-*}+\tau\left((-)^{(n+1) r}: C_{r} \rightarrow C_{r}\right)} \\
& =\alpha_{n}\left(C^{n-*}\right)+(-)^{n} \alpha_{n}(C)^{*}+(n+1) \sum_{r \text { odd }} \epsilon\left(C_{r}, C_{r}\right) \\
& =\sum_{r \equiv n+2, n+3(\bmod 4)}\left(\epsilon\left(C_{r}, C_{r}\right)+\epsilon\left(C_{n-r}, C_{n-r}\right)\right) \\
& +(n+1) \sum_{r \text { odd }} \epsilon\left(C_{r}, C_{r}\right) \\
& = \begin{cases}0 & \text { for } n \equiv 0 \text { or } n \equiv 3 \\
\epsilon(\chi(C), \chi(C)) & \text { for } n \equiv 1 \text { or } n \equiv 2\end{cases} \\
& =\frac{n}{2}(n+1) \epsilon(\chi(C), \chi(C)) \text {. }
\end{aligned}
$$

This proves the Lemma.

Lemma 19 The torsion of a contractible signed chain complex $C$ in $A$ satisfies

$$
\tau^{\mathrm{NEW}}\left(C^{n-*}\right)=(-)^{n+1} \tau^{\mathrm{NEW}}(C)^{*} \in K_{1}^{\mathrm{iso}}(\mathbb{A}) .
$$

Proof We denote by $\bar{C}^{n-*}$ the chain complex with $\left(\bar{C}^{n-*}\right)_{r}=\left(C_{n-r}\right)^{*}$ and

$$
d_{\bar{C}^{n-*}}=d_{C}^{*}: \bar{C}^{n-r} \rightarrow \bar{C}^{n-r+1} .
$$

We have an isomorphism $f: C_{r}^{n-*} \rightarrow \bar{C}_{r}^{n-*}$ given by $f=-1$ if $r \equiv n+2, n+3(\bmod 4)$ and $f=1$ otherwise. By considering the torsion of this isomorphism we have

$$
\tau\left(C^{n-*}\right)=\tau\left(\bar{C}^{n-*}\right)+\alpha_{n}(C) .
$$

Let $n_{\text {even }}$ be the greatest even integer $\leq n$, similarly $n_{\text {odd }}$. For any chain contraction $\Gamma$ for $C$ we have the following commutative diagram:




The torsion of the lower map in this diagram is $\tau(C)^{*}$; the torsion of the uppermost map is $(-)^{n+1} \tau\left(\bar{C}^{n-*}\right)$. So, by first considering the torsions of the maps in the above diagram we have

$$
\begin{aligned}
\tau\left(\bar{C}^{n-*}\right) & =(-)^{n+1} \tau(C)^{*}+(-)^{n+1}\left(\sum_{i>j ; i, j \text { even }} \epsilon\left(C^{i}, C^{j}\right)-\sum_{i>j ; i, j \text { odd }} \epsilon\left(C^{i}, C^{j}\right)\right) \\
& =(-)^{n+1} \tau(C)^{*}+(-)^{n} \beta(C, C)^{*} .
\end{aligned}
$$

Hence by (3-1)

$$
\tau\left(C^{n-*}\right)=(-)^{n+1} \tau(C)^{*}+(-)^{n} \beta(C, C)^{*}+\alpha_{n}(C) .
$$

Using the definition of the dual signed chain complex we have

$$
\tau^{\mathrm{NEW}}\left(C^{n-*}\right)=(-)^{n+1} \tau^{\mathrm{NEW}}(C)^{*} .
$$

Lemma 20 Let $C, D$ be $n$-dimensional signed chain complexes in $\mathbb{A}$ and $f: C \rightarrow D$ a chain equivalence. Then

$$
\tau^{\mathrm{NEW}}\left(f^{n-*}: D^{n-*} \rightarrow C^{n-*}\right)=(-)^{n} \tau^{\mathrm{NEW}}(f)^{*} \in K_{1}^{\mathrm{iso}}(\mathbb{A}) .
$$

Proof We have an isomorphism of chain complexes $\theta: \mathcal{C}\left(f^{n-*}\right) \rightarrow \mathcal{C}(f)^{n+1-*}$ given by

$$
\mathcal{C}\left(f^{n-*}\right)_{r}=C^{n-r} \oplus D^{n-r+1} \stackrel{\left(\begin{array}{c}
0(-)^{n-r} \\
1
\end{array}\right)}{\longrightarrow} \mathcal{C}(f)_{r}^{n+1-*}=D^{n-r+1} \oplus C^{n-r} .
$$

The torsion of the map $\theta$ is given by

$$
\begin{aligned}
\tau^{\mathrm{NEW}}(\theta)= & \tau^{\mathrm{NEW}}\left((-)^{n-r}: S\left(D^{n-*}\right) \rightarrow D^{n+1-*}\right) \\
& +\tau^{\mathrm{NEW}}\left(C^{n-*} \rightarrow(S C)^{n+1-*}\right) \\
& +\tau^{\mathrm{NEW}}\left((S C)^{n+1-*} \oplus D^{n+1-*} \rightarrow(S C \oplus D)^{n+1-*}\right) \\
& +(-)^{n+1} \tau^{\mathrm{NEW}}(D \oplus S C \rightarrow S C \oplus D)^{*} \\
= & n \epsilon(\chi(D), \chi(D))^{*}+(n+1) \epsilon(\chi(D), \chi(D))^{*} \\
& +\epsilon(\chi(D), \chi(D))^{*} \\
= & 0
\end{aligned}
$$

and the result follows since $\tau^{\mathrm{NEW}}\left(\mathcal{C}(f)^{n+1-*}\right)=(-)^{n} \tau^{\mathrm{NEW}}(f)^{*}$.

We define the duality isomorphism $T$ as

$$
T: \operatorname{Hom}_{\AA}\left(C^{p}, D_{q}\right) \rightarrow \operatorname{Hom}_{\AA}\left(D^{q}, C_{p}\right) ; \phi \rightarrow(-)^{p q} \phi^{*} .
$$


Lemma 21 Let $C, D$ be $n$-dimensional signed chain complexes in $\mathbb{A}$ and $f: C^{n-*} \rightarrow$ $D$ a chain equivalence. Then

$$
\tau^{\mathrm{NEW}}\left(T f: D^{n-*} \rightarrow C\right)=(-)^{n} \tau^{\mathrm{NEW}}(f)^{*}+\frac{n}{2}(n+1) \epsilon(\chi(C), \chi(C)) \in K_{1}^{\mathrm{iso}}(\mathbb{A}) .
$$

Proof Using Lemma 18 and Lemma 20 we have

$$
\begin{aligned}
\tau^{\mathrm{NEW}}\left(T f: D^{n-*} \rightarrow C\right)= & \tau^{\mathrm{NEW}}\left(f^{*}: D^{n-*} \rightarrow\left(C^{n-*}\right)^{n-*}\right) \\
& +\tau^{\mathrm{NEW}}\left((-1)^{(n+1) r}\left(C^{n-*}\right)^{n-*} \rightarrow C\right) \\
= & (-)^{n} \tau^{\mathrm{NEW}}(f)^{*}+\frac{n}{2}(n+1) \epsilon(\chi(C), \chi(C))
\end{aligned}
$$

as required.

Together the above three lemmas prove Proposition 16.

\section{Torsion of Poincaré complexes}

We now move on to consider symmetric Poincaré complexes. These are algebraic objects which encapsulate the properties of Poincaré duality spaces (see Ranicki [5; 6] for a more complete discussion). We will restrict ourselves to considering symmetric Poincaré complexes over a ring $R$, that is we work over $\mathbb{A}=\mathbb{A}(R)$ and we will consider the torsion invariants to lie in the more familiar $K_{1}(R)$. We will define the notion of the absolute torsion of a symmetric Poincaré complex to be, essentially, the torsion of the Poincaré duality chain equivalence. In the case of compact oriented manifolds $M^{n}$ with $C W$-structure this is the torsion of the map

$$
\left[M^{n}\right] \cap-: C(M)^{n-*} \rightarrow C(M) .
$$

In this section we develop the theory from this algebraic viewpoint; it will be applied to geometric objects in a later section.

We recall from Ranicki $[5 ; 9]$ the following definition.

\section{Definition 22}

(1) An $n$-dimensional symmetric complex $\left(C, \phi_{0}\right)$ is a chain complex $C$ in $\mathbb{A}(R)$, together with a collection of morphisms

$$
\phi=\left\{\phi_{s}: C^{n-r+s} \rightarrow C_{r} \mid s \geq 0\right\}
$$

such that

$$
d_{C} \phi_{s}+(-)^{r} \phi_{s} d_{C}^{*}+(-)^{n+s+1}\left(\phi_{s-1}+(-)^{s} T \phi_{s-1}\right)=0: C^{n-r+s-1} \rightarrow C_{r}
$$


where $s \geq 0$, and $\phi_{-1}=0$. Hence $\phi_{0}: C^{n-*} \rightarrow C$ is a chain map and $\phi_{1}$ is a chain homotopy $\phi_{1}: \phi_{0} \simeq T \phi_{0}$.

(2) The complex is said to be Poincaré if $\phi_{0}$ is a chain equivalence.

(3) The complex is said to be round if $C$ is round.

(4) A morphism between $n$-dimensional symmetric complexes $(C, \phi)$ and $\left(C^{\prime}, \phi^{\prime}\right)$ consists of a chain map $f: C \rightarrow C^{\prime}$ and morphisms $\sigma_{s}: C^{\prime n+1+s-r} \rightarrow C_{r}^{\prime} s \geq 0$ such that

$\phi_{s}^{\prime}-f \phi_{s} f^{*}=d_{C^{\prime}} \sigma_{s}+(-)^{r} \sigma_{s} d_{C^{\prime}}^{*}+(-)^{n+s}\left(\sigma_{s-1}+(-)^{s} T \sigma_{s-1}\right): C^{\prime n-r+s} \rightarrow C_{r}^{\prime}$

(in particular $\phi_{0}^{\prime} \simeq f \phi_{0} f^{*}$ ). Such a morphism is said to be a homotopy equivalence if $f$ is a chain equivalence.

(5) A symmetric complex $(C, \phi)$ is said to be connected if $H_{0}\left(\phi_{0}: C^{n-*} \rightarrow C\right)=0$.

(6) The boundary $(\partial C, \partial \phi)$ of a connected $n$-dimensional symmetric complex $(C, \phi)$ is the $(n-1)$-dimensional symmetric Poincaré complex defined by

$$
\begin{aligned}
& d_{\partial C}=\left(\begin{array}{cc}
d_{C} & (-)^{r} \phi_{0} \\
0 & (-)^{r} d_{C}^{*}
\end{array}\right): \partial C_{r}=C_{r+1} \oplus C^{n-r} \longrightarrow \partial C_{r-1}=C_{r} \oplus C^{n+1-r} \\
& \partial \phi_{0}=\left(\begin{array}{cc}
(-)^{n-r-1} T \phi_{1} & (-)^{r n} \\
1 & 0
\end{array}\right): \partial C^{n-r-1}=C^{n-r} \oplus C_{r+1} \longrightarrow \partial C_{r}=C_{r+1} \oplus C^{n-r} \\
& \partial \phi_{S}=\left(\begin{array}{cc}
(-)^{n-r-1} T \phi_{s+1}(-)^{r n} \\
0
\end{array}\right): \partial C^{n-r+s-1}=C^{n+s-r} \oplus C_{r-s+1} \longrightarrow \\
& \quad \partial C_{r}=C_{r+1} \oplus C^{n-r} .
\end{aligned}
$$

(7) A signed symmetric (Poincaré) complex is a symmetric (Poincaré) complex $\left(C, \phi_{0}\right)$ where in addition $C$ is a signed chain complex.

Example 23 An $n$-dimension manifold $M$ with universal covering $\widetilde{M}$ determines a symmetric Poincaré complex $(C(\widetilde{M}), \phi)$ in $\mathbb{A}\left(\mathbf{Z}\left[\pi_{1} M\right]\right)$ with

$$
\phi_{0}=[M] \cap-: C(\widetilde{M})^{n-*} \rightarrow C(\widetilde{M}) .
$$

Lemma 24 The boundary $(\partial C, \partial \phi)$ of any signed $n$-dimensional symmetric complex $(C, \phi)$ satisfies

$$
\tau^{\mathrm{NEW}}\left(\partial \phi_{0}:(\partial C)^{n-1-*} \rightarrow \partial C\right)=\frac{n}{2}(n+1) \epsilon(\chi(C), \chi(C)) \in K_{1}(R) .
$$

Proof The map

$$
\partial \phi_{0}=\left(\begin{array}{cc}
(-)^{n-r-1} T \phi_{1} & (-)^{r n} \\
1 & 0
\end{array}\right): \partial C^{n-r-1} \rightarrow \partial C_{r}
$$

is an isomorphism. 
We have

$$
\begin{aligned}
\tau^{\mathrm{NEW}}\left(\partial \phi_{0}\right)= & \tau^{\mathrm{NEW}}\left(\left(\begin{array}{cc}
0 & (-)^{r n} \\
1 & 0
\end{array}\right):\left(\Omega C \oplus C^{n-*}\right)^{n-1-*} \rightarrow \Omega C \oplus C^{n-*}\right) \\
= & \tau^{\mathrm{NEW}}\left(\left(\Omega C \oplus C^{n-*}\right)^{n-1-*} \rightarrow(\Omega C)^{n-1-*} \oplus\left(C^{n-*}\right)^{n-1-*}\right) \\
& +\tau^{\mathrm{NEW}}\left(\left(C^{n-*}\right)^{n-1-*} \rightarrow\left(\Omega C^{n-1-*}\right)^{n-1-*}\right) \\
& +\tau^{\mathrm{NEW}}\left((-)^{n r}:\left(\Omega C^{n-1-*}\right)^{n-1-*} \rightarrow \Omega C\right) \\
& +\tau^{\mathrm{NEW}}\left((\Omega C)^{n-1-*} \rightarrow C^{n-*}\right) \\
= & \tau^{\mathrm{NEW}}\left(C^{n-*} \oplus \Omega C \rightarrow \Omega C \oplus C^{n-*}\right) \\
= & \frac{n}{2}(n+1) \epsilon(\chi(C), \chi(C))
\end{aligned}
$$

using the results of Lemma 18.

We can now define a new absolute torsion invariant of Poincaré complexes which is additive and a cobordism invariant.

Definition 25 We define the absolute torsion of a signed Poincaré complex $(C, \phi)$ as

$$
\tau^{N E W}(C, \phi)=\tau^{\mathrm{NEW}}\left(\phi_{0}\right) \in K_{1}(R) .
$$

Proposition 26 Let $(C, \phi)$ and $\left(C^{\prime}, \phi^{\prime}\right)$ be signed $n$-dimensional Poincaré complexes.

Then:

(1) Additivity:

$$
\tau^{N E W}\left(C \oplus C^{\prime}, \phi \oplus \phi^{\prime}\right)=\tau^{N E W}(C, \phi)+\tau^{N E W}\left(C^{\prime} \phi_{0}^{\prime}\right) \in K_{1}(R) .
$$

(2) Duality:

$$
\tau^{N E W}(C, \phi)=(-)^{n} \tau^{N E W}(C, \phi)^{*}+\frac{n}{2}(n+1) \epsilon(\chi(C), \chi(C)) \in K_{1}(R)
$$

(n.b. the above sign term disappears in the case where anti-symmetric forms over the ring $R$ necessarily have even rank; this is the case for $R=\mathbf{Z}$ or $R=\mathbf{Q}$ but not $R=\mathbb{C}$ ).

(3) Homotopy invariance: Suppose $\left(f, \sigma_{s}\right)$ is a homotopy equivalence from $(C, \phi)$ to $\left(C^{\prime}, \phi^{\prime}\right)$. Then

$$
\tau^{\mathrm{NEW}}\left(C^{\prime}, \phi^{\prime}\right)=\tau^{\mathrm{NEW}}(C, \phi)+\tau(f)+(-)^{n} \tau(f)^{*} \in K_{1}(R) .
$$


(4) Cobordism Invariance: Suppose that $(C, \phi)$ is homotopy equivalent to the boundary of some $(n+1)$-dimensional symmetric complex with torsion $\left(D, \phi^{D}\right)$. Then

$$
\begin{aligned}
\tau^{N E W}(C, \phi)= & (-)^{n+1} \tau^{\mathrm{NEW}}(C \rightarrow \partial D)^{*}-\tau^{\mathrm{NEW}}(C \rightarrow \partial D) \\
& +\frac{1}{2}(n+1)(n+2) \epsilon(\chi(D), \chi(D)) \in K_{1}(R) .
\end{aligned}
$$

(5) Orientation change:

$$
\tau^{N E W}(C,-\phi)=\tau^{N E W}(C, \phi)+\epsilon(\chi(C), \chi(C)) \in K_{1}(R) .
$$

(6) The absolute torsion of a signed Poincaré complex is independent of the choice of sign $\eta_{C}$.

\section{Proof}

(1) A symmetric Poincaré complex of odd dimension satisfies $\chi(C)=0$, hence the map $\left(C \oplus C^{\prime}\right)^{n-*} \rightarrow C^{n-*} \oplus C^{\prime n-*}$ has trivial absolute torsion. Additivity now follows from the additivity of chain equivalences.

(2) We know that $\phi_{0}$ is homotopic to $T \phi_{0}$; duality now follows by applying Lemma 21.

(3) We have that $\phi_{0}^{\prime} \simeq f \phi_{0} f^{*}$ and hence

$$
\tau^{\mathrm{NEW}}\left(\phi_{0}^{\prime}\right)=\tau^{\mathrm{NEW}}(f)+\tau^{\mathrm{NEW}}\left(\phi_{0}\right)+(-)^{n} \tau^{\mathrm{NEW}}(f)^{*} .
$$

(4) This follows from Lemma 24 and homotopy invariance.

(5) We have that $\tau^{\mathrm{NEW}}\left(-\phi_{0}\right)=\tau^{\mathrm{NEW}}\left(\phi_{0}\right)+\tau^{\mathrm{NEW}}(-1: C \rightarrow C)=\tau^{\mathrm{NEW}}\left(\phi_{0}\right)+$ $\chi(C) \tau(-1)$.

(6) A change in $\eta_{C}$ leads to a corresponding change in $\eta_{c^{n-*}}$ so $\tau^{\mathrm{NEW}}\left(\phi_{0}\right)$ is unchanged.

\section{The signed Poincaré derived category with involution}

In this section we will add an involution to a particular subcategory of the signed derived category. Let $\mathbb{S P D} \mathbb{D}_{n}(\mathbb{A}(R))$ denote the category whose object are signed $n-$ dimensional chain complexes $C$ in $\mathbb{A}(R)$ which are isomorphic to their dual complexes $C^{n-*}$ and $\chi(C)=0$ if $n$ is odd. Then we have an involution

$$
\begin{aligned}
*: C & \mapsto C^{n-*} \\
*:(f: C \rightarrow D) & \mapsto\left(f^{n-*}: D^{n-*} \rightarrow C^{n-*}\right)
\end{aligned}
$$


with the natural equivalence $e(C)$ given by

$$
e(C)=(-)^{(n+1) r}: C \rightarrow\left(C^{n-*}\right)^{n-*} .
$$

We call this category the signed Poincaré derived category with n-involution. In order to show that this is a covariant functor of additive categories we must show that $*(A \oplus B)=* A \oplus * B$. However, the condition that $\chi(C)$ is odd if $n$ is odd implies that the torsion of the rearrangement map $(C \oplus D)^{n-*} \rightarrow C^{n-*} \oplus D^{n-*}$ is trivial (see Lemma 18 part (2)) and the functor $*$ is additive. As in the case of the signed derived category we have a map

$$
i_{*}: K_{1}^{\text {iso }}\left(\mathbb{S P D}_{n}(\mathbb{A}(R))\right) \rightarrow K_{1}^{\text {iso }}(\mathbb{A}(R)) ; \tau^{i s o}(f) \mapsto \tau^{\mathrm{NEW}}(f) .
$$

The behaviour of $i_{*}$ under the involution on $\mathbb{S P D}_{n}(\mathbb{A}(R))$ is given by

$$
i_{*}\left(f^{*}\right)=(-)^{n} i_{*}(f)^{*} .
$$

\section{A product formula}

In this section we will quote a formula for the absolute torsion of a product of symmetric Poincaré complexes and prove it in a special case.

All tensor products will be over the integers $\mathbf{Z}$. For rings $R$ and $R^{\prime}$ we have natural inclusion maps $K_{1}(R) \rightarrow K_{1}\left(R \otimes R^{\prime}\right)$ and $K_{1}\left(R^{\prime}\right) \rightarrow K_{1}\left(R \otimes R^{\prime}\right)$. For an isomorphism $f: A \rightarrow B$ in $\mathbb{A}(R)$ and a module $M$ in $\mathbb{A}\left(R^{\prime}\right)$ we have

$$
\begin{aligned}
& \tau(f \otimes 1: A \otimes M \rightarrow B \otimes M)=\operatorname{rank}_{R^{\prime}}(M) \tau(f) \in K_{1}\left(R \otimes R^{\prime}\right) \\
& \tau(1 \otimes f: M \otimes A \rightarrow M \otimes B)=\operatorname{rank}_{R^{\prime}}(M) \tau(f) \in K_{1}\left(R \otimes R^{\prime}\right) .
\end{aligned}
$$

We recall from [5] the definition of the tensor product of symmetric Poincaré complexes.

\section{Definition 27}

(1) The tensor product $C \otimes D$ of a chain complex $C$ in $\mathbb{A}(R)$ and a chain complex $D$ in $\mathbb{A}\left(R^{\prime}\right)$ is the chain complex in $\mathbb{A}\left(R \otimes R^{\prime}\right)$

$$
\begin{aligned}
d_{C \otimes D}:(C \otimes D)_{r}=\sum_{s=-\infty}^{\infty} C_{s} \otimes D_{r-s} & \rightarrow(C \otimes D)_{r-1} ; \\
x \otimes y & \mapsto x \otimes d_{D}(y)+(-)^{r-s} d_{C}(x) \otimes y .
\end{aligned}
$$


(2) The tensor product $(C \otimes D, \phi \otimes \theta)$ of an $n$-dimensional symmetric Poincaré complex $(C, \phi)$ with an $m$-dimensional symmetric Poincaré complex $(D, \theta)$ is an $(n+m)$-dimensional Poincaré complex defined by

$$
(\phi \otimes \theta)_{s}=\sum_{r=-\infty}^{s}(-1)^{(n+r) s} \phi_{r} \otimes T \theta_{s-r}:(C \otimes D)^{n+m-*} \rightarrow C \otimes D .
$$

Proposition 28 Let $(C, \phi)$ and $\left(C^{\prime}, \phi^{\prime}\right)$ be symmetric Poincaré complexes over a rings with involution $R$ and $R^{\prime}$ respectively. Then

$$
\tau^{\mathrm{NEW}}\left(C \otimes C^{\prime}, \phi \otimes \phi^{\prime}\right)=\chi(C) \tau^{\mathrm{NEW}}\left(C^{\prime}, \phi^{\prime}\right)+\chi\left(C^{\prime}\right) \tau^{\mathrm{NEW}}(C, \phi) .
$$

The proof of this result requires the theory of signed complexes to be extended to tensor products; this theory is developed in [1] using the theory of signed filtered complexes.

We will use the following "ad hoc" methods to prove the product formula under the following conditions.

Assumption 29 The rings $R$ and $R^{\prime}$ are such that $(4 k+2)$-dimensional symmetric forms necessarily have even rank (eg group rings).

The ring $R=\mathbb{C}$ is an example which does not satisfy this assumption. We say a module $M$ in $\mathbb{A}(R)$ is even if $\operatorname{rank}_{R}(M)$ is even; similarly we say a chain complex $C$ in $\mathbb{A}(R)$ is even if $C_{r}$ is even for all $r$.

Definition 30 Let $\left(C, \eta_{C}\right)$ and $\left(D, \eta_{D}\right)$ be even signed complexes. We define the signed complex tensor product by

$$
\left(C \otimes D, \eta_{C \otimes D}\right)=(C \otimes D, 0) .
$$

\section{Lemma 31}

(1) Let $C, C^{\prime}$ be signed, even complexes in $\mathbb{A}(R)$ and $D$ a signed even complex in $\mathbb{A}\left(R^{\prime}\right)$. Then

$$
\begin{aligned}
& \tau^{\mathrm{NEW}}\left(\left(C \oplus C^{\prime}\right) \otimes D \rightarrow(C \otimes D) \oplus\left(C^{\prime} \otimes D\right)\right)=0 \\
& \tau^{\mathrm{NEW}}\left(D \otimes\left(C \oplus C^{\prime}\right) \rightarrow(D \otimes C) \oplus\left(D \otimes C^{\prime}\right)\right)=0 .
\end{aligned}
$$

(2) Let $C$ be a signed contractible even complex in $\mathbb{A}(R)$ and $D$ a signed even complex in $\mathbb{A}\left(R^{\prime}\right)$. Then

$$
\tau^{\mathrm{NEW}}(C \otimes D)=\tau^{\mathrm{NEW}}(D \otimes C)=\chi(D) \tau^{\mathrm{NEW}}(C) .
$$


(3) Let $f: C \rightarrow C^{\prime}$ be a chain equivalence of even complexes in $\mathbb{A}(R)$ and $D$ a signed even complex in $\mathbb{A}\left(R^{\prime}\right)$. Then

$$
\begin{aligned}
\tau^{\mathrm{NEW}}\left(f \otimes 1: C \otimes D \rightarrow C^{\prime} \otimes D\right) & =\tau^{\mathrm{NEW}}\left(1 \otimes f: D \otimes C \rightarrow D \otimes C^{\prime}\right) \\
& =\chi(D) \tau^{\mathrm{NEW}}(f) .
\end{aligned}
$$

Proof For even modules $M, N$ the map $\epsilon(M, N)=0 \in K_{1}(R)$ and $\tau(-1: M \rightarrow$ $M)=0$, so the torsion of rearrangement maps is always zero and $\eta_{C \oplus D}=\eta_{D}+\eta_{C}$ for even chain complexes $C, D$. Part 1 follows straight from these facts. For part 2, let $\Gamma: C_{*} \rightarrow C_{*+1}$ be a chain contraction of $C$, then $\left(d_{C}+\Gamma\right) \otimes 1$ is a chain contraction of $C \otimes D$. We have a commutative diagram



with the bottom map given by $\left(d_{C}+\Gamma\right) \otimes 1 \oplus\left(d_{C}+\Gamma\right) \otimes 1$. The torsion of the top map is $\tau(C \otimes D)$, the torsion of the bottom map is $\chi(D) \tau(C)$. Part 2 follows from the fact that the torsions of the left and right maps are zero, since they are rearrangements. For part 3 we have that

$$
\tau^{\mathrm{NEW}}(\mathcal{C}(f \otimes 1) \rightarrow \mathcal{C}(f) \otimes D)=0
$$

since it is a rearrangement map and the result now follows straight from part 2.

Proposition 32 Let $\left(C, \phi^{C}\right)$ and $\left(D, \phi^{D}\right)$ be Poincaré complexes over ring $R$ and $R^{\prime}$ respectively, where $R$ and $R^{\prime}$ satisfy Assumption 29. Then

$$
\begin{aligned}
\tau^{\mathrm{NEW}}\left(C \otimes D, \phi^{C} \otimes \phi^{D}\right)= & \chi(D) \tau^{\mathrm{NEW}}\left(C, \phi^{C}\right)+\chi(C) \tau^{\mathrm{NEW}}\left(D, \phi^{D}\right) \\
& \in K_{1}\left(R \otimes R^{\prime}\right) .
\end{aligned}
$$

Proof Given any f.g. based chain complex $C$ we may form a direct sum with a contractible chain complex to form a new chain complex which is of even rank in every dimension except one. Hence given a Poincaré pair $(C, \phi)$ we may form a new Poincaré pair $\left(C^{\prime}, \phi^{C^{\prime}}\right)$ which is even in every dimension by

(1) Forming the direct sum with a contractible complex (letting $\phi^{C^{\prime}}$ vanish on this contractible complex) such that $C^{\prime}$ is even in every dimension except possibly the middle. 
(2) If the complex is odd (and hence of dimension $4 \mathrm{k}$ ), forming the direct sum with the Poincaré complex which is $R$ in dimension $2 k$, vanishes otherwise and has $\phi_{0}=1: R \rightarrow R$.

We may form a similar complex $\left(D^{\prime}, \phi^{D^{\prime}}\right)$ from $\left(D, \phi^{D}\right)$. Using Lemma 31 we see that

$$
\tau^{\mathrm{NEW}}\left(\phi_{0}^{C^{\prime}} \otimes \phi_{0}^{D^{\prime}}\right)=\chi\left(C^{\prime}\right) \tau^{\mathrm{NEW}}\left(\phi_{0}^{D^{\prime}}\right)+\chi\left(D^{\prime}\right) \tau^{\mathrm{NEW}}\left(\phi_{0}^{C^{\prime}}\right) \in K_{1}\left(R \otimes R^{\prime}\right) .
$$

Hence

$$
\tau^{\mathrm{NEW}}\left(C^{\prime} \otimes D^{\prime}, \phi^{C^{\prime}} \otimes \phi^{D^{\prime}}\right)=\chi\left(C^{\prime}\right) \tau^{\mathrm{NEW}}\left(D^{\prime}, \phi_{0}^{D^{\prime}}\right)+\chi\left(D^{\prime}\right) \tau^{\mathrm{NEW}}\left(C^{\prime}, \phi_{0}^{C^{\prime}}\right) .
$$

Let $R^{C}$ denote the chain complex which is 0 if $C$ is even and $R$ in dimension $2 k$ otherwise; similarly $R^{\prime} D$. By direct computation (for the sake of clarity we now suppress mention of the morphisms $\phi$ )

$$
\begin{aligned}
\tau^{\mathrm{NEW}}\left(R^{C} \otimes D\right) & \left.=\chi(C)\left(\tau^{\mathrm{NEW}}(D)+(k+1) \chi(D)\right) \tau(-1)\right) \\
\tau^{\mathrm{NEW}}\left(C \otimes{R^{\prime}}^{D}\right) & =\chi(D)\left(\tau^{\mathrm{NEW}}(C)+(l+1) \chi(C) \tau(-1)\right) \\
\tau^{\mathrm{NEW}}\left(R^{C} \otimes{R^{\prime}}^{D}\right) & =(k+l) \chi(C) \chi(D) \tau(-1) .
\end{aligned}
$$

The Poincaré complex $\left(C^{\prime} \otimes D^{\prime}\right)$ is homotopy equivalent to $\left(C \oplus R^{C}\right) \otimes\left(D \oplus R^{\prime}\right)$; using the invariance of absolute torsion under homotopy equivalence, its additivity properties and the above three formulae we have

$$
\tau^{\mathrm{NEW}}(C \otimes D)=\chi(C) \tau^{\mathrm{NEW}}(D)+\chi(D) \tau^{\mathrm{NEW}}(C) \in K_{1}\left(R \otimes R^{\prime}\right)
$$

as required.

\section{Round L-theory}

We refer the reader to [2] for the definition of the round symmetric $L$-groups $L_{r}^{n}(A)$. The absolute torsion defined in this paper as $\tau\left(C, \phi_{0}\right)=\tau\left(\phi_{0}\right)$ (here $\tau\left(\phi_{0}\right)$ refers to the absolute torsion defined in [7]) is not a cobordism invariant. We can define such an invariant using the absolute torsion of a Poincare complex. If this invariant is substituted for $\tau\left(C, \phi_{0}\right)$ as defined in [2] then the results become correct.

Lemma 33 Let $(C, \phi)$ be a round Poincaré complex. The reduced element

$$
\tau^{\mathrm{NEW}}(C, \phi) \in \widehat{H}^{n}\left(\mathbf{Z}_{2} ; K_{1}(R)\right)
$$


is independent of the choice of sign $\eta_{C}$; moreover we have a well defined homomorphism

$$
L_{r}^{n}(R) \rightarrow \widehat{H}^{n}\left(\mathbf{Z}_{2} ; K_{1}(R)\right)
$$

given by $(C, \phi) \mapsto \tau^{\mathrm{NEW}}(C, \phi)$.

Proof The element $\tau^{\mathrm{NEW}}(C, \phi) \in \widehat{H}^{n}\left(\mathbf{Z}_{2} ; K_{1}(R)\right)$ is independent of the choice of sign by Proposition 26 part (6). The absolute torsion is additive by Proposition 26 part (1). The absolute torsion of the boundary of a round symmetric complex is trivial in the reduced group $\widehat{H}^{n}\left(\mathbf{Z}_{1} ; K_{1}(R)\right)$ by Proposition 26 part (4). Hence the torsion of a round null-cobordant complex is trivial and the map

$$
L_{r}^{n}(R) \rightarrow \widehat{H}^{n}\left(\mathbf{Z}_{2} ; K_{1}(R)\right)
$$

given by $(C, \phi) \mapsto \tau^{\mathrm{NEW}}(C, \phi)$ is well defined.

\section{Applications to manifolds}

\subsection{The absolute torsion of oriented manifolds}

To any $n$-dimensional oriented manifold $M$ we may associate a Poincaré complex $(C, \phi)$ over the ring $R=\mathbf{Z}\left[\pi_{1} M\right]$, well defined up to homotopy equivalence (see [6]). By property (2) of Proposition 26 the absolute torsion of such a Poincaré complex satisfies

$$
\tau^{\mathrm{NEW}}(C, \phi)=(-)^{n} \tau^{\mathrm{NEW}}(C, \phi)^{*}
$$

since $\chi(M) \equiv 0(\bmod 2)$ unless $n \equiv 0(\bmod 4)$. Hence the torsion $\tau^{\mathrm{NEW}}(C, \phi)$ may be considered to lie in the group $\widehat{H}^{n}\left(\mathbf{Z}_{2} ; K_{1}\left(\mathbf{Z}\left[\pi_{1} M\right]\right)\right)$. By property (3) of Proposition 26 if $(C, \phi)$ is homotopy equivalent to $\left(C^{\prime}, \phi^{\prime}\right)$ then

$$
\tau^{\mathrm{NEW}}(C, \phi)=\tau^{\mathrm{NEW}}\left(C^{\prime}, \phi^{\prime}\right) \in \widehat{H}^{n}\left(\mathbf{Z}_{2} ; K_{1}\left(\mathbf{Z}\left[\pi_{1} M\right]\right)\right)
$$

hence

$$
\tau^{\mathrm{NEW}}\left(M^{n}\right):=\tau^{\mathrm{NEW}}(C, \phi) \in \widehat{H}^{n}\left(\mathbf{Z}_{2} ; \mathbf{Z}\left[\pi_{1} M\right]\right)
$$

is well defined.

\subsection{Examples of the absolute torsion of manifolds}

8.2.1 The circle We may associate to the circle $\left(S^{1}\right)$ the following chain complex over $R=\mathbf{Z}\left[\pi_{1}\left(S^{1}\right)\right]=\mathbf{Z}\left[t, t^{-1}\right]$ by giving it the $\mathrm{CW}$-decomposition consisting of one 
1-cell and one 0-cell:

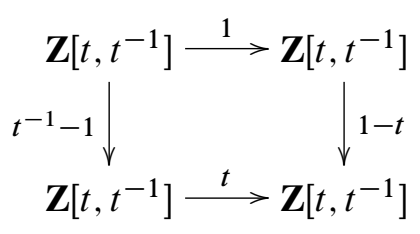

In this diagram the two modules on the right are the chain complex, the two modules on the left are the dual complex and the sideways arrows represent $\phi_{0}$.

Hence $\tau^{\mathrm{NEW}}\left(S^{1}\right)=\tau(-t) \in \widehat{H}^{n}\left(\mathbf{Z}_{2} ; K_{1}\left(\mathbf{Z}\left[t, t^{-1}\right]\right)\right.$.

8.2.2 The absolute torsion of an algebraic mapping torus The mapping torus of a map $f: M \rightarrow M$ is the space obtained from $M \times I$ by attaching the boundaries $M \times\{0\}$ and $M \times\{1\}$ using the map $f$. The following algebraic analogue is defined by Ranicki [10, Definition 24.3] (the reader should note the different sign convention used here).

Definition 34 The algebraic mapping torus of a morphism $(f, \sigma):(C, \phi) \rightarrow(C, \phi)$ from an $n$-dimensional symmetric Poincaré complex $(C, \phi)$ over a ring $R$ to itself is the $(n+1)$-dimensional symmetric complex $(T(f), \theta)$ over the ring $R\left[z, z^{-1}\right]$ defined by

$$
\begin{aligned}
T(f) & =\mathcal{C}(f-z) \\
\theta_{s} & =\left(\begin{array}{cc}
(-)^{n} \sigma_{s} & (-)^{s} \phi_{s} z \\
(-)^{n-r+1} \phi_{s} f^{*}(-)^{n-r+s+1} T \phi_{s-1}
\end{array}\right): T(f)^{n-r+s+1} \rightarrow T(f)_{r} .
\end{aligned}
$$

The complex is Poincare if the chain map $f$ is a chain equivalence.

Lemma 35 Let $(f, \sigma):(C, \phi) \rightarrow(C, \phi)$ be a self chain equivalence from an $n-$ dimensional symmetric Poincaré complex $(C, \phi)$ over a ring $R$ to itself. Then

$$
\tau^{\mathrm{NEW}}(T(f), \theta)=\tau^{\mathrm{NEW}}(f)+\chi(C) \tau(-z) \in K_{1}\left(R\left[z, z^{-1}\right]\right) .
$$

Proof We have a commutative diagram with short exact rows:

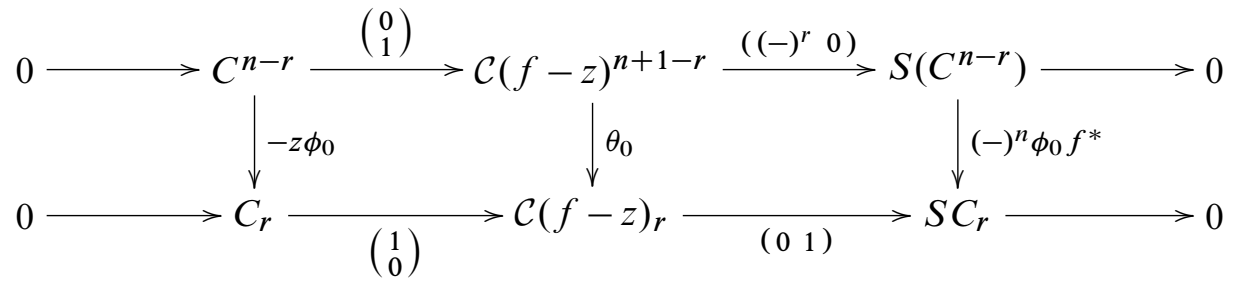


The absolute torsion of the lower short exact sequence is trivial; for the top map we have

$$
\begin{aligned}
& \tau^{\mathrm{NEW}}\left(C^{n-r}, \mathcal{C}(f-z)^{n+1-r}, S\left(C^{n-r}\right) ;\left(\begin{array}{l}
0 \\
1
\end{array}\right),\left((-)^{r} 0\right)\right) \\
& =\tau^{\mathrm{NEW}}\left(\left(\begin{array}{cc}
0 & (-)^{r} \\
1 & 0
\end{array}\right):(C \oplus S C)^{n+1-*} \rightarrow C^{n-*} \oplus S\left(C^{n-*}\right)\right) \\
& =\tau^{\mathrm{NEW}}\left((C \oplus S C)^{n+1-*} \rightarrow C^{n+1-*} \oplus(S C)^{n+1-*}\right) \\
& \quad+\tau^{\mathrm{NEW}}\left((-1)^{r}: C^{n+1-*} \rightarrow S\left(C^{n-*}\right)\right) \\
& \quad+\tau^{\mathrm{NEW}}\left((S C)^{n+1-*} \rightarrow C^{n-*}\right) \\
& \quad+\tau^{\mathrm{NEW}}\left(S\left(C^{n-*}\right) \oplus C^{n-*} \rightarrow C^{n-*} \oplus S\left(C^{n-*}\right)\right) \\
& =0 .
\end{aligned}
$$

Using Proposition 13 part (4) we have

$$
\begin{aligned}
\tau^{\mathrm{NEW}}(T(f), \theta) & =\tau^{\mathrm{NEW}}\left(\theta_{0}\right) \\
& =\tau^{\mathrm{NEW}}\left(-z \phi_{0}\right)+\tau^{\mathrm{NEW}}\left((-1)^{n} S\left(\phi_{0} f^{*}\right): S\left(C^{n-*}\right) \rightarrow S C\right) \\
& =-\tau^{\mathrm{NEW}}\left(f^{*}\right)+\tau^{\mathrm{NEW}}(-z: C \rightarrow C) \\
& =(-)^{n+1-*} \tau^{\mathrm{NEW}}(f)^{*}+\tau^{\mathrm{NEW}}(-z: C \rightarrow C) \\
& =\tau^{\mathrm{NEW}}(f)+\chi(C) \tau(-z)
\end{aligned}
$$

as required.

8.2.3 A specific example of a mapping torus We return to the example of the orientation preserving self-homeomorphism $f: \mathbb{C P}^{2} \rightarrow \mathbb{C P}^{2}$ given by complex conjugation in some choice of homogeneous coordinates (see Section 1.6). We recall that the torsion of this map is $\tau^{\mathrm{NEW}}(f)=\tau(-1) \in K_{1}(\mathbf{Z})$. Using Lemma 35 we compute the absolute torsion of the mapping torus of $f$ as

$$
\tau^{\mathrm{NEW}}(T(f))=\tau\left(z^{3}\right) \in K_{1}\left(\mathbf{Z}\left[z, z^{-1}\right]\right)
$$

where $z$ is a generator of $\pi_{1}(T(f))=\pi_{1}\left(S^{1}\right)=\mathbf{Z}$. By contrast we may compute the absolute torsion of the space $T\left(I d: \mathbb{C P}^{2} \rightarrow \mathbb{C P}^{2}\right)=S^{1} \times \mathbb{C P}^{2}$ as

$$
\tau^{\mathrm{NEW}}\left(S^{1} \times \mathbb{C P}^{2}\right)=\tau\left(-z^{3}\right) \in K_{1}\left(\mathbf{Z}\left[z, z^{-1}\right]\right)
$$

hence the absolute torsion can distinguish between these two $\mathbb{C P}^{2}$ bundles over $S^{1}$. A more thorough investigation into the absolute torsion of fibre bundles of compact manifolds is made in [1]. 


\section{Identifying the sign term}

Throughout this section we work over a group ring $R=\mathbf{Z}[\pi]$ for some group $\pi$ (or, more generally, any ring with involution $R$ which admits a map $R \rightarrow \mathbf{Z}$ such that the composition $\mathbf{Z} \rightarrow R \rightarrow \mathbf{Z}$ is the identity). We first identify the relationship between the "sign" term of the absolute torsion of a Poincare complex and the traditional signature and Euler characteristic and semi-characteristic invariants.

We have a canonical decomposition of $K_{1}(\mathbf{Z}[\pi])$ as follows:

$$
K_{1}(\mathbf{Z}[\pi])=\widetilde{K}_{1}(\mathbf{Z}[\pi]) \oplus \mathbf{Z}_{2}
$$

with the $\mathbf{Z}_{2}$ component the "sign" term identified by the map

$$
i_{*} K_{1}(\mathbf{Z}[\pi]) \rightarrow K_{1}(\mathbf{Z})=\mathbf{Z}_{2}
$$

induced by the augmentation map $i: \pi \rightarrow 1$ (more generally, a map $R \rightarrow \mathbf{Z}$ gives a map $K_{1}(R) \rightarrow K_{1}(\mathbf{Z})=\mathbf{Z}_{2}$ which gives a splitting $\left.K_{1}(R)=\widetilde{K}_{1}(R) \oplus \mathbf{Z}_{2}\right)$. We wish to determine the $\mathbf{Z}_{2}$ component in terms of more traditional invariants of Poincaré complexes. The augmentation map may also be applied to a symmetric complex $(C, \phi)$ over $\mathbf{Z}[\pi]$ to form a symmetric complex over $\mathbf{Z}$ by forgetting the group. Functoriality of the absolute torsion tells us that this complex has the same sign term as $(C, \phi)$, hence to identify the sign term it is sufficient to consider symmetric Poincaré complexes over $\mathbf{Z}$. We will require the Euler semi-characteristic $\chi_{1 / 2}(C)$ of Kervaire [3]

Definition 36 The Euler semi-characteristic $\chi_{1 / 2}(C)$ of a $(2 k-1)$-dimensional chain complex $C$ over a field $F$ is defined by

$$
\chi_{1 / 2}(C)=\sum_{i=0}^{k-1}(-)^{i} \operatorname{rank}_{F} H_{i}(C) \in \mathbf{Z}
$$

For a $(2 k-1)$-dimensional chain complex $C$ over $\mathbf{Z}$ we define

$$
\chi_{1 / 2}(C ; F)=\chi_{1 / 2}(C \otimes \mathbf{z} F) .
$$

Proposition 37 The absolute torsion of an $n$-dimensional symmetric Poincaré complex over $\mathbf{Z}$ is determined by the signature and the Euler characteristic and semicharacteristic as follows:

(1) If $n=4 k$ then

$$
\tau^{\mathrm{NEW}}(C, \phi)=\frac{1}{2}(\sigma(C)-(1+2 k) \chi(C)) \tau(-1)
$$

with $\sigma(C)$ the signature of the complex. 
(2) If $n=4 k+1$ then $\tau^{\mathrm{NEW}}(C, \phi)=\chi_{1 / 2}(C ; \mathbf{Q})$.

(3) Otherwise $\tau^{\mathrm{NEW}}(C, \phi)=0$.

As an example we have a simple corollary.

Corollary 38 The absolute torsion of an orientation preserving self-homeomorphism of a simply-connect manifold of dimension $4 k+2$ is trivial.

Proof Let $f: M \rightarrow M$ be such a self-homeomorphism. We may triangulate $M$ and hence construct the algebraic mapping torus $T(f)$ of the chain equivalence $f: C(M) \rightarrow C(M)$. By Lemma 35

$$
\tau^{\mathrm{NEW}}(T(f))=\tau^{\mathrm{NEW}}(f) \in K_{1}\left(\mathbf{Z}\left[z, z^{-1}\right]\right) .
$$

The augmentation map $\epsilon: \mathbf{Z} \rightarrow 1$ induces a map of rings $\epsilon_{*}: \mathbf{Z}\left[z, z^{-1}\right] \rightarrow \mathbf{Z}$. Since $L$-theory and the absolute torsion are functorial, $\epsilon_{*} T(f)$ represents an element of $L^{4 k+3}(\mathbf{Z})$ with absolute torsion $\tau^{\mathrm{NEW}}\left(\epsilon_{*} T(f)\right)=\tau^{\mathrm{NEW}}(f) \in K_{1}(\mathbf{Z})$. However by part 3 of the above proposition $\tau^{\mathrm{NEW}}\left(\epsilon_{*} T(f)\right)=0$.

The aim of the rest of this section is to prove Proposition 37 . We recall from [6] the computation of the symmetric $L$-groups $L_{h}^{n}(\mathbf{Z})$ of the integers $\mathbf{Z}$ :

$$
L_{h}^{n}(\mathbf{Z})= \begin{cases}\mathbf{Z}(\text { signature }) & n \equiv 0(\bmod 4) \\ \mathbf{Z}_{2}(\text { de Rham invariant }) & n \equiv 1(\bmod 4) \\ 0 & n \equiv 2(\bmod 4) \\ 0 & n \equiv 3(\bmod 4)\end{cases}
$$

The deRham invariant $d(C) \in Z_{2}$ of a $(4 k+1)$-dimensional Poincaré complex was expressed in Lusztig-Milnor-Peterson [4] as the difference

$$
d(C)=\chi_{1 / 2}\left(C ; \mathbf{Z}_{2}\right)-\chi_{1 / 2}(C ; \mathbf{Q}) .
$$

For dimensions $n \equiv 2,3(\bmod 4)$ the absolute torsion is a cobordism invariant (Proposition 26 part (4)) so the above computation of the symmetric $L$-groups tells us that the absolute torsion is trivial in these cases, thus proving the third part of Proposition 37.

If $n=4 k+1$ then the absolute torsion is not a cobordism invariant; however it is a round cobordism invariant, so absolute torsion defines a map

$$
L_{r h}^{4 k+1}(\mathbf{Z}) \rightarrow K_{1}(\mathbf{Z}) .
$$


Since $\chi(C)=0$ for all odd-dimensional symmetric Poincaré complexes every such $(4 k+1)$-dimensional complex represents an element in $L_{r h}^{4 k+1}(\mathbf{Z})$. In [2, Proposition 4.2] the group $L_{r h}^{4 k+1}(\mathbf{Z})$ is identified as

$$
L_{r h}^{4 k+1}(\mathbf{Z})=\mathbf{Z}_{2} \oplus \mathbf{Z}_{2} ; C \mapsto\left(\chi_{1 / 2}\left(C ; \mathbf{Z}_{2}\right), \chi_{1 / 2}(C, \mathbf{Q})\right) .
$$

We now construct explicit generators of this group and compute their absolute torsions. We define the generator $(G, \phi)$ to have chain complex $G$ concentrated in dimensions $2 k$ and $2 k+1$ defined by

$$
d_{G}=0: G_{2 k+1}=\mathbf{Z} \rightarrow G_{2 k}=\mathbf{Z}
$$

with the morphisms $\phi$ given by

$$
\phi_{0}=\left\{\begin{array}{l}
1: G^{2 k}=\mathbf{Z} \rightarrow G_{2 k+1}=\mathbf{Z} \\
1: G^{2 k+1}=\mathbf{Z} \rightarrow G_{2 k}=\mathbf{Z}
\end{array} \phi_{1}=0 .\right.
$$

Geometrically $(G, \phi)$ is the symmetric Poincaré complex over $\mathbf{Z}$ associated to the circle. By direct computation, $\chi_{1 / 2}\left(G, \mathbf{Z}_{2}\right)=1, \chi_{1 / 2}(G, \mathbf{Q})=1$ and $\tau^{\mathrm{NEW}}(G, \phi)=\tau(-1)$. We define the generator $(H, \psi)$ to have chain complex $H$ concentrated in dimensions $2 k$ and $2 k+1$ defined by

$$
d_{H}=2: H_{2 k+1}=\mathbf{Z} \rightarrow H_{2 k}=\mathbf{Z},
$$

with the morphisms $\psi$ given by

$$
\psi_{0}=\left\{\begin{array}{l}
-1: H^{2 k}=\mathbf{Z} \rightarrow H_{2 k+1}=\mathbf{Z} \\
1: H^{2 k+1}=\mathbf{Z} \rightarrow H_{2 k}=\mathbf{Z}
\end{array} \psi_{1}=1: H^{2 k+1} \rightarrow H_{2 k+1} .\right.
$$

Geometrically $(H, \psi)$ is a symmetric Poincaré complex over $\mathbf{Z}$ which is cobordant to the complex associated to the mapping torus of the self-diffeomorphism of $\mathbf{C P}^{2}$ given by complex conjugation. Again by direct computation, $\chi_{1 / 2}\left(H, \mathbf{Z}_{2}\right)=1, \chi_{1 / 2}(H, \mathbf{Q})=0$ and $\tau^{\mathrm{NEW}}(H, \psi)=0$. By considering the absolute torsion of these two generators we see that the map $L_{r h}^{4 k+1}(\mathbf{Z}) \rightarrow K_{1}(\mathbf{Z})$ is given by

$$
(C, \phi) \mapsto \chi_{1 / 2}(C, \mathbf{Q}) \tau(-1)
$$

thus proving part two of Proposition 37.

To prove part 1 of Proposition 37 we use the following lemma taken from [1].

Lemma 39 We have the following relationship between $\tau^{\mathrm{NEW}}$ and signature modulo 4 of a $4 k$-dimensional Poincaré complex $(C, \phi)$

$$
\sigma(C)=2 \tau^{\mathrm{NEW}}(C, \phi)+(2 k+1) \chi(C) \in \mathbf{Z}_{4}
$$

where the map 2: $K_{1}(\mathbf{Z})=\mathbf{Z}_{2} \rightarrow \mathbf{Z}_{4}$ takes $\tau(-1)$ to $2 \in \mathbf{Z}_{4}$. 
A simple rearrangement of the formula of the above lemma yields the first part of Proposition 37.

\section{References}

[1] I Hambleton, A Korzeniewski, A Ranicki, The signature of a fibre bundle is multiplicative mod 4, Geom. Topol. 11 (2007) 251-314

[2] I Hambleton, A Ranicki, L Taylor, Round L-theory, J. Pure Appl. Algebra 47 (1987) 131-154 MR906966

[3] M Kervaire, Courbure intégrale généralisée et homotopie, Math. Ann. 131 (1956) 219-252 MR0086302

[4] G Lusztig, J Milnor, F P Peterson, Semi-characteristics and cobordism, Topology 8 (1969) 357-359 MR0246308

[5] A Ranicki, The algebraic theory of surgery. I. Foundations, Proc. London Math. Soc. (3) 40 (1980) 87-192 MR560997

[6] A Ranicki, The algebraic theory of surgery. II. Applications to topology, Proc. London Math. Soc. (3) 40 (1980) 193-283 MR566491

[7] A Ranicki, The algebraic theory of torsion. I. Foundations, from: "Algebraic and geometric topology (New Brunswick, N.J., 1983)", Lecture Notes in Math. 1126, Springer, Berlin (1985) 199-237 MR802792

[8] A Ranicki, Additive L-theory, K-Theory 3 (1989) 163-195 MR1029957

[9] A A Ranicki, Algebraic L-theory and topological manifolds, Cambridge Tracts in Mathematics 102, Cambridge University Press, Cambridge (1992) MR1211640

[10] A Ranicki, High-dimensional knot theory, Springer Monographs in Mathematics, Springer, New York (1998) MR1713074

School of Mathematics, University of Edinburgh

Edinburgh EH9 3JZ, United Kingdom

ajkorzeniewski@yahoo.co.uk

Proposed: Tom Goodwillie

Received: 20th April 2005

Seconded: Wolfgang Lueck and Steve Ferry

Accepted: 6th January 2007 